\title{
Optimization of One-Step Block Method for Solving Second-Order Fuzzy Initial Value Problems
}

\author{
Safa Al-Refai $\mathbb{D}^{1},{ }^{1}$ Muhammed I. Syam $\mathbb{D}^{1},{ }^{1}$ and Mohammed Al-Refai $\mathbb{D}^{2}$ \\ ${ }^{1}$ Department of Mathematical Sciences College of Science, UAE University Al-Ain, Al Ain, UAE \\ ${ }^{2}$ Department of Mathematics Yarmouk University, Irbid, Jordan \\ Correspondence should be addressed to Muhammed I. Syam; m.syam@uaeu.ac.ae
}

Received 24 December 2020; Revised 29 March 2021; Accepted 9 April 2021; Published 29 April 2021

Academic Editor: Ning Cai

Copyright (c) 2021 Safa Al-Refai et al. This is an open access article distributed under the Creative Commons Attribution License, which permits unrestricted use, distribution, and reproduction in any medium, provided the original work is properly cited.

In this article, we present a one-step hybrid block method for approximating the solutions of second-order fuzzy initial value problems. We prove the stability and convergence results of the method and present several examples to illustrate the efficiency and accuracy of the proposed method. The numerical results are compared with the existing ones in the literature.

\section{Introduction}

The second-order initial value problems (2IVPs) of the form

$$
y^{\prime \prime}=f\left(t, y, y^{\prime}\right), y(a)=y_{0}, y^{\prime}(a)=y_{1}, \quad t \in[a, b],
$$

where $f$ is a continuous function on $[a, b]$, are interesting problems and they have many applications, particularly in engineering, physics, biology, and chemistry fields. In general, it is very difficult to find the exact solution to such problems especially when $f$ is a nonlinear function of $t, y$, and $y^{\prime}$. Therefore, numerical methods can be used to find their approximate solutions. There are several numerical methods to solve these problems such as the one-step block method [1, 2], Taylor series method [3], Adomian decomposition method [4], fourth-order and Butcher's fifth-order Runge-Kutta methods [5], and the two-step hybrid block method [6]. In this paper, we are interested to study the 2IVPs of a fuzzy type. These problems consist of fuzzy differential equations (FDEs) with fuzzy initial conditions. The fuzzy initial value problems (FIVPs) are often incomplete or ambiguous. For instance, initial conditions or the values of fuzzy differential equations may not be known accurately. In this situation, FDEs appear as a natural way to model dynamical systems under possibilities of uncertainty. To solve these equations, we define the derivative by one of three different approaches (see [7]). The first approach is based on the Hukuhara derivative instituted by Puri-Ralescu in 1983. The second approach is known as Zadeh's extension principle, and the last approach is strongly generalized differentiability which is presented by Bede and Gal in 2005. In this study, we focus on the Hukuhara derivative in order to define our differential equations. The FIVPs have several applications that have been highlighted in many research areas, such as civil engineering, physics, control theory, economics, population models, and modeling hydraulic [8-10]. Most problems in physics and engineering are modeled by initial value problems (IVPs). They have many applications such as Bagley-Torvik problem [11], Lane-Emden second-order equations [12], and delay IVP [13]. Since the exact solution for such problems is difficult to compute, several researchers use numerical methods to deal with this task. For example, Hossain et al. [5] used the Runge-Kutta method of order four, Ramos et al. [6] used the hybrid block method (HBM), and Jameel et al [14] used the homotopy analysis method (HAM) and the optimum homotopy analysis method (OHAM).

Second-order IVPs play an important role in several applications. Rufai et al. [12] solved the Lane-Emden second-order singular differential equations using three off-step points. They did not implement the second-order IVP. The block system they got is different from our block method. They combined the HBM with an appropriate algorithm 
which is applied to the first subinterval to circumvent the singular behavior at the left endpoint of the integration interval which made his accuracy depend on both methods. Also, they integrated part of the equation to deal with the singularity in his problem.

We consider the fuzzy second-order initial value problems of the form

$$
y^{\prime \prime}=f\left(t, y, y^{\prime}\right), y(a)=\widehat{a}, y^{\prime}(a)=\widehat{b}, \quad t \in[a, b],
$$

where $\widehat{a}$ and $\widehat{b}$ are fuzzy numbers and $f$ is the fuzzy function. Since the function and initial conditions are fuzzy, we apply the $\beta$-level set operations to obtain the components of the problem which are as follows:

$$
\begin{aligned}
(y(t))_{\beta}= & \left(y_{1, \beta}(t), y_{2, \beta}(t)\right),(\widehat{a})_{\beta}=\left(a_{1}, a_{2}\right),(\widehat{b})_{\beta}=\left(b_{1}, b_{2}\right), \\
\left(y^{\prime}(t)\right)_{\beta}= & \left(y_{1, \beta}^{\prime}(t), y_{2, \beta}^{\prime}(t)\right),\left(y^{\prime \prime}(t)\right)_{\beta}=\left(y_{1, \beta}^{\prime \prime}(t), y_{2, \beta}^{\prime \prime}(t)\right), \\
\left(f\left(t, y, y^{\prime}\right)\right)_{\beta}= & \left(f_{1}\left(t,\left(y_{1, \beta}(t), y_{2, \beta}(t)\right),\left(y_{1, \beta}^{\prime}(t), y_{2, \beta}^{\prime}(t)\right)\right),\right. \\
& \left.f_{2}\left(t,\left(y_{1, \beta}(t), y_{2, \beta}(t)\right),\left(y_{1, \beta}^{\prime}(t), y_{2, \beta}^{\prime}(t)\right)\right)\right), \\
f_{1}= & \min \left\{f\left(t, w, w^{\prime}\right): w \in\left(y_{1, \beta}(t), y_{2, \beta}(t)\right), w^{\prime} \in\left(y_{1, \beta}^{\prime}(t), y_{2, \beta}^{\prime}(t)\right)\right\}, \\
f_{2}= & \max \left\{f\left(t, w, w^{\prime}\right): w \in\left(y_{1, \beta}(t), y_{2, \beta}(t)\right), w^{\prime} \in\left(y_{1, \beta}^{\prime}(t), y_{2, \beta}^{\prime}(t)\right)\right\} .
\end{aligned}
$$

We then solve the above min-max problems directly. These kinds of problems might be difficult to solve directly and obtaining exact solutions is not always possible. Therefore, researchers were interested in obtaining numerical solutions by using different methods, such as the decomposition method [15], the homotopy analysis method $[14,16]$, the Runge-Kutta method [17, 18], the least-square method [19], the interactive and standard arithmetic [20-22], the Fréchet derivative method [23], and solving delay fuzzy problems [24]. For more references, see $[25,26,33-36]$.

The decomposition method is investigated in many papers (see [15]). However, it does not work for all types of problems (see [15]). We use the direct method (min-max problem) to find the solution for the problems in [15], and it works for some of them. However, the direct method is sometimes very complicated and it may not be possible to apply it. Thus, we proposed a new method to find numerical solutions for these problems. Our method depends on the one-step hybrid block method. In this method, we try to optimize the local truncation errors in order to find the best choice of the step point. The main advantage of the proposed method is that it is self-starter where we do not need to use other methods to generate more initial starting conditions.

The HBM is easy to use and it gives accurate results. We implement it for the second-order fuzzy initial value problems and it shows that the method works accurately. We use only one off-step method and it gives better results than other methods such as HAM and OHAM. This will open a new door for the researchers to use this approach to solve such problems. Its computational cost is small compared to other methods in the literature review. The order of the proposed method is 3 and the method is stable and convergent.

The current paper is organized as follows. In Section 2, preliminaries of fuzzy concepts and theorems will be presented. In Sections 3 and 4, we present the optimized one- step hybrid block method and some theoretical results. In Section 5, we apply the proposed method to the fuzzy initial value problems of second order, and we present some numerical results in Section 6 to show the efficiency of the proposed method. Finally, in Section 7, the results will be discussed and some conclusions will be presented.

\section{Preliminaries}

In this section, some preliminaries will be presented to be used in this paper.

Definition 1 (see [27]). A fuzzy number is a function $\psi: \mathbb{R} \longrightarrow[0,1]$ that satisfies the following:

(1) There exists $c \in \mathbb{R}$ such that $\psi(c)=1$

(2) $\psi(s a+(1-s) b) \geq \min \{\psi(a), \psi(b)\}$ for all $a, b \in \mathbb{R}$ and $s \in[0,1]$

(3) The set $\{x \in \mathbb{R}: \psi(x) \geq \beta\}$ is a closed subset of $\mathbb{R}$ for all $\beta \in(0,1]$

(4) The closure of $\{x \in \mathbb{R}: \psi(x)>0\}$ is a compact set

The set of all fuzzy numbers is denoted by $F_{\mathbb{R}}$. If $\psi \in F_{\mathbb{R}}$ and $\beta \in(0,1]$, the $\beta$-level set is given by

$$
\psi_{\beta}=\{x \in \mathbb{R}: \psi(x) \geq \beta\},
$$

and the 0 -level set is given by

$$
\psi_{0}=\operatorname{closure}\{x \in \mathbb{R}: \psi(x)>0\} .
$$

It is easy to see that we can write $\psi_{\beta}=\left[\underline{\psi}_{\beta}, \bar{\psi}_{\beta}\right]$, where it holds that

(1) $\psi_{\beta} \subseteq \psi_{\gamma}$ if $0<\gamma \leq \beta \leq 1$

(2) If the sequence $\left\{\beta_{n}\right\}$ is an increasing sequence in $(0,1]$ converging to $\beta$, then $\operatorname{Lim}_{n \longrightarrow \infty} \psi_{\beta_{n}}=\psi_{\beta}$

(3) For any $\beta \in(0,1],-\infty<\underline{\psi}_{\beta} \leq \bar{\psi}_{\beta}<\infty$ 
Example 1. Let $\psi=\left(a_{1}, a_{2}, a_{3}\right)$ be a triangular fuzzy number. Then,

$$
\psi(s)= \begin{cases}\frac{s-a_{1}}{a_{2}-a_{1}}, & a_{1} \leq s \leq a_{2}, \\ \frac{s-a_{3}}{a_{2}-a_{3}}, & a_{2} \leq s \leq a_{3}, \\ 0, & \text { otherwise, }\end{cases}
$$

and $\psi_{\beta}=\left[(1-\beta) a_{1}+\beta a_{2},(1-\beta) a_{3}+\beta a_{2}\right]$. The graph of the symmetric fuzzy triangle $\psi=(0,1,2)$ is given in Figure 1.

Definition 2 (see [27]). Let $\widehat{a}, \widehat{b} \in F_{\mathbb{R}}, \beta \in[0,1]$, and $c \in \mathbb{R}$ where $\widehat{a}_{\beta}=\left[\underline{a}_{\beta}, \bar{a}_{\beta}\right]$ and $\widehat{b}_{\beta}=\left[\underline{b}_{\beta}, \bar{b}_{\beta}\right]$. Then,

(1) $(\hat{a} \oplus \widehat{b})_{\beta}=\left[\underline{a}_{\beta}+\underline{b}_{\beta}, \bar{a}_{\beta}+\bar{b}_{\beta}\right]$,

(2) $(\hat{a} \ominus \widehat{b})_{\beta}=\left[\underline{a}_{\beta}-\bar{b}_{\beta}, \bar{a}_{\beta}-\underline{b}_{\beta}\right]$,

(3) $(c \odot \widehat{a})_{\beta}= \begin{cases}{\left[c \underline{a}_{\beta}, c \bar{a}_{\beta}\right],} & c \geq 0, \\ {\left[c \bar{a}_{\beta}, c \underline{a}_{\beta}\right],} & c<0,\end{cases}$

(4) If there exists $\widehat{c} \in F_{\mathbb{R}}$ such that $\widehat{a}=\widehat{b}+\widehat{c}$, then $\widehat{c}=$ $\widehat{a} \ominus_{H} \widehat{b}$ is the $H$-difference of $\widehat{a}$ and $\widehat{b}$

(5) The Hausdorff matrix $\Gamma_{H}: F_{\mathbb{R}} \times F_{\mathbb{R}} \longrightarrow[0, \infty)$ is defined by

$$
\Gamma_{H}(\widehat{a}, \widehat{b})=\sup _{0<b \leq 1}\left\{\max \left\{\left|\underline{a}_{\beta}-\underline{b}_{\beta}\right|,\left|\bar{a}_{\beta}-\bar{b}_{\beta}\right|\right\}\right\}
$$

and $\left(F_{\mathbb{R}}, \Gamma_{H}\right)$ is a matrix space.

Definition 3 (see [7]). The fuzzy-valued function is a function $\widehat{h}: V \longrightarrow F_{\mathbb{R}}$, where $V$ be a real vector space and $F_{\mathbb{R}}$ be the set of fuzzy numbers. The function $\widehat{h}(x)$ can be written as $\left[f_{\beta}(x), g_{\beta}(x)\right]$ for all $x \in V$ and any $\beta \in[0,1]$. The functions $f_{\beta}(x)$ and $g_{\beta}(x)$ are called $\beta$-cut functions of the fuzzy-valued function $\widehat{h}$.

Definition 4 (see [7]). Let $f:(a, b) \subset \mathbb{R} \longrightarrow F_{\mathbb{R}}$ be a fuzzy function and $x \in(a, b)$. If there exists $f^{\prime}(x) \in F_{\mathbb{R}}$ such that

$$
f^{\prime}(x)=\lim _{\Delta \longrightarrow 0^{+}} \frac{f(x+\Delta) \ominus_{H} f(x)}{\Delta}=\lim _{\Delta \longrightarrow 0^{+}} \frac{f(x) \ominus_{H} f(x-\Delta)}{\Delta},
$$

then $f$ is called Hukuhara differentiable at $x$ and the Hukuhara derivative of $f$ at $x$ is $f^{\prime}(x)$.

Theorem 1 (see [28]). Let $\sigma \in F_{\mathbb{R}}$ and $g:(a, b) \subset \mathbb{R}^{+} \longrightarrow$ $(0, \infty)$ be differentiable at $x_{0} \in(a, b)$. Let $f:(a, b) \longrightarrow F_{\mathbb{R}}$ be a fuzzy function defined by $f(x)=\sigma \odot g(x)$. If $g^{\prime}\left(x_{0}\right)>0$, then $f$ is Hukuhara differentiable at $x_{0}$ and the Hukuhara derivative of $f$ at $x_{0}$ is $f^{\prime}\left(x_{0}\right)=\sigma \odot g^{\prime}\left(x_{0}\right)$.

Example 2. Let $\sigma \in F_{\mathbb{R}}$ and $f: \mathbb{R} \longrightarrow F_{\mathbb{R}}$ be a fuzzy function defined by $f(x)=\sigma \odot(x+1)$. Then, $g(x)=x+1$. If $x>-1$, then

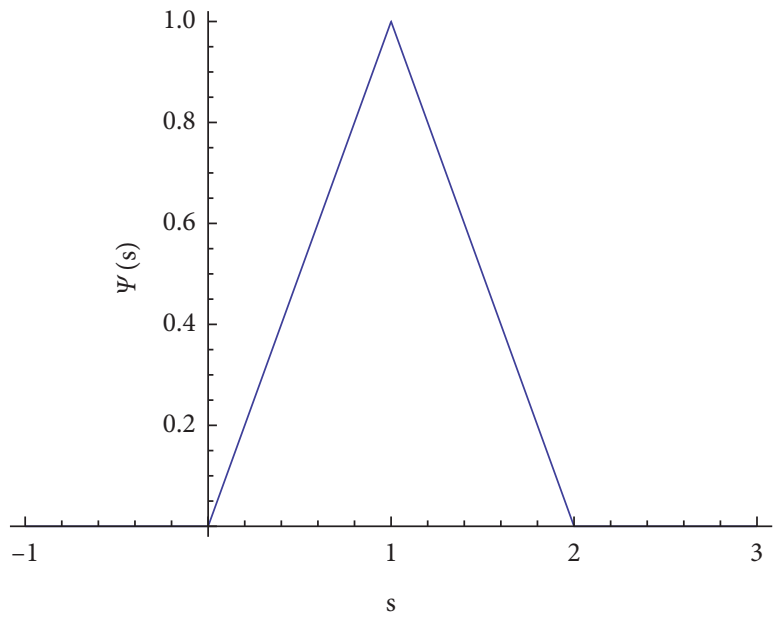

Figure 1: Fuzzy triangle number $\psi=(0,1,2)$.

$$
\begin{aligned}
& g(x)=x+1>0, \\
& g^{\prime}(x)=1>0 .
\end{aligned}
$$

Using Theorem 1, we have $f$ is Hukuhara differentiable on $(0, \infty)$ and the Hukuhara derivative of $f$ is $f^{\prime}(x)=\sigma \odot 1$. For $x<-1, \Delta>0$, but close to 0 , and $\beta \in(0,1]$, we have

$$
\begin{aligned}
& \underline{f}_{\beta}(x+\Delta)-\underline{f}_{\beta}(x)=\Delta \bar{\sigma}_{\beta}, \\
& \bar{f}_{\beta}(x+\Delta)-\bar{f}_{\beta}(x)=\Delta \underline{\sigma}_{\beta},
\end{aligned}
$$

where $f=[f, \bar{f}]$. Since

$$
\underline{f}_{\beta}(x+\Delta)-\underline{f}_{\beta}(x) \not \bar{f}_{\beta}(x+\Delta)-\bar{f}_{\beta}(x),
$$

then $f(x+\Delta) \ominus_{H} f(x)$ does not exist. Thus, $f$ is not Hukuhara differentiable on $(-\infty, 0)$. For $x=-1, \Delta>0$, but close to 0 , and $\beta \in(0,1]$, we have

$$
f_{\beta}= \begin{cases}{\left[\underline{\sigma}_{\beta}(x+1), \bar{\sigma}_{\beta}(x+1)\right],} & x>-1, \\ {\left[\bar{\sigma}_{\beta}(x+1), \underline{\sigma}_{\beta}(x+1)\right],} & x \leq-1,\end{cases}
$$

which implies that $f$ is not Hukuhara differentiable at $x=0$.

\section{One-Step Hybrid Block Method with One Off- Step Point}

In this section, we drive a numerical method based on the one-step hybrid block method with one off-step point (HBM1), $t_{n+k}$ with $0<k<1$, to solve the following differential equation of the form

$$
\begin{aligned}
& y^{\prime \prime}(t)=f\left(t, y(t), y^{\prime}(t)\right), \quad t \geq 0, \\
& y\left(t_{0}\right)=y_{0}, \\
& y^{\prime}\left(t_{0}\right)=y_{1} .
\end{aligned}
$$

To derive HBM1, we assume that $t_{n}=n h$ where $h$ is the step size. Since we are planning to use one-step hybrid method with one off-step point, we need to interpolate the 
solution and its first derivative at $t_{n}$ and to collocate the IVP at $t_{n}$ and $t_{n+k}$. Then, we solve these equations for the coefficients of the approximate solution. Thus, we have five equations. To able to get a unique solution, we assume that the solution has five coefficients. For this reason, we approximate the solution by polynomial of degree four. If we increase the order of the polynomial, we should take more off-step points. We approximate the solution of problem (13)-(15) by a polynomial of degree 4 as follows:

$$
y(t) \simeq \sum_{j=0}^{4} a_{j} t^{j}
$$

and its first derivative by

$$
y^{\prime}(t) \simeq \sum_{j=1}^{4} j a_{j} t^{j-1}
$$

and its second derivative by

$$
y^{\prime \prime}(t) \simeq \sum_{j=2}^{4} j(j-1) a_{j} t^{j-2}
$$

Interpolate equations (16) and (17) at the point $t_{n}$ and collocate equation (18) at the points $t_{n}, t_{n+k}=t_{n}+k h$, and $t_{n+1}=t_{n}+h$ to get the following system:

$$
\left(\begin{array}{ccccc}
1 & t_{n} & t_{n}^{2} & t_{n}^{3} & t_{n}^{4} \\
0 & 1 & 2 t_{n} & 3 t_{n}^{2} & 4 t_{n}^{3} \\
0 & 0 & 2 & 6 t_{n} & 12 t_{n}^{2} \\
0 & 0 & 2 & 6 t_{n+k} & 12 t_{n+k}^{2} \\
0 & 0 & 2 & 6 t_{n+1} & 12 t_{n+1}^{2}
\end{array}\right)\left(\begin{array}{c}
a_{0} \\
a_{1} \\
a_{2} \\
a_{3} \\
a_{4}
\end{array}\right)=\left(\begin{array}{c}
y_{n} \\
y_{n}^{\prime} \\
f_{n} \\
f_{n+k} \\
f_{n+1}
\end{array}\right)
$$

where $y_{n+j} \approx y\left(t_{n+j}\right), y_{n+j}^{\prime} \approx y^{\prime}\left(t_{n+j}\right)$, and $f_{n+j} \approx y^{\prime \prime}\left(t_{n+j}\right)$, $j=0, k, 1$. Solving system (19) after substituting $t=t_{n}+w h$ to get

$$
\begin{aligned}
y(w) & \approx y_{n}+h \alpha y_{n}^{\prime}+h^{2}\left(\alpha_{0} f_{n}+\alpha_{k} f_{n+k}+\alpha_{1} f_{n+1}\right), \\
y^{\prime}(w) & \approx \beta y_{n}^{\prime}+h\left(\beta_{0} f_{n}+\beta_{k} f_{n+k}+\beta_{1} f_{n+1}\right),
\end{aligned}
$$

where $\alpha, \alpha_{0}, \alpha_{k}, \alpha_{1}, \beta, \beta_{0}, \beta_{k}$, and $\beta_{1}$ are functions of $w$. We then evaluate the approximation of $y(w)$ and $y^{\prime}(w)$ at $w=k$ and 1 , to get

$$
\begin{aligned}
& y_{n+j}=y_{n}+h \alpha_{j} y_{n}^{\prime}+h^{2}\left(\alpha_{0, j} f_{n}+\alpha_{k, j} f_{n+k}+\alpha_{1, j} f_{n+1}\right) \\
& y_{n+j}^{\prime}=\beta_{j} y_{n}^{\prime}+h\left(\beta_{0, j} f_{n}+\beta_{k, j} f_{n+k}+\beta_{1, j} f_{n+1}\right)
\end{aligned}
$$

where $j=k, 1$, and

$$
\begin{aligned}
& \alpha_{1}=1, \alpha_{0,1}=\frac{1}{3}-\frac{1}{12 k}, \alpha_{k, 1}=\frac{1}{12 k-12 k^{2}}, \alpha_{1,1}=\frac{1}{12}\left(2+\frac{1}{k-1}\right), \\
& \alpha_{k}=k, \alpha_{0, k}=-\frac{1}{12}(k-4) k^{2}, \alpha_{k, k}=\frac{(k-2) k^{2}}{12(k-1)}, \alpha_{1, k}=\frac{k^{4}}{12(k-1)}, \\
& \beta_{1}=1, \beta_{0,1}=\frac{1}{2}-\frac{1}{6 k}, \beta_{k, 1}=\frac{1}{6 k-6 k^{2}}, \beta_{1,1}=\frac{1}{6}\left(3+\frac{1}{k-1}\right), \\
& \beta_{k}=1, \beta_{0, k}=-\frac{1}{6}(k-3) k, \beta_{k, k}=\frac{(2 k-3) k}{6(k-1)}, \beta_{1, k}=\frac{k^{3}}{6(k-1)} .
\end{aligned}
$$

In the literature review, researchers used a uniform partition to the interval $[0,1]$ which makes the method of order 2. However, in this paper, we will choose the partition which makes the method have the largest possible order. To do that, we leave $k$ as a parameter and we choose it so that the proposed method has the largest possible order. To maximize the order of the implicit block method (21) when $j=1$, we minimize the local truncation errors in the formula for $y_{n+1}$,

$$
\mathscr{L}\left(y\left(t_{n+1}\right) ; h\right)=\frac{5 k-2}{360} h^{5} y^{(5)}\left(t_{n}\right)+\frac{\left(5 k^{2}+5 k-3\right)}{1440} h^{6} y^{(6)}\left(t_{n}\right)+\mathcal{O}\left(h^{7}\right) .
$$

To maximize the order, we solve the following equation for $k$ where $0<k<1$

$$
\frac{5 k-2}{360}=0
$$

Hence, 


$$
k=\frac{2}{5}
$$

and the local truncation errors for $y_{n+1}, y_{n+k}, y_{n+1}^{\prime}$, and $y_{n+k}^{\prime}$ are

$$
\begin{aligned}
& \mathscr{L}\left[y\left(t_{n+1}\right) ; h\right]=-\frac{h^{6} y^{(6)}\left(t_{n}\right)}{7200}+\mathcal{O}\left(h^{7}\right)=-1.38889 * 10^{-4} h^{6} y^{(6)}\left(t_{n}\right)+\mathcal{O}\left(h^{7}\right), \\
& \mathscr{L}\left[y\left(t_{n+k}\right) ; h\right]=\frac{14 h^{5} y^{(5)}\left(t_{n}\right)}{46875}+\mathcal{O}\left(h^{6}\right)=2.98667 * 10^{-4} h^{5} h^{5} y^{(5)}\left(t_{n}\right)+\mathcal{O}\left(h^{6}\right), \\
& \mathscr{L}\left[y^{\prime}\left(t_{n+1}\right) ; h\right]=-\frac{h^{5} y^{(5)}\left(t_{n}\right)}{360}+\mathcal{O}\left(h^{5}\right)=-2.7777 * 10^{-3} h^{5} y^{(5)}\left(t_{n}\right)+\mathcal{O}\left(h^{6}\right), \\
& \mathscr{L}\left[y^{\prime}\left(t_{n+k}\right) ; h\right]=\frac{8 h^{5} y^{(5)}\left(t_{n}\right)}{5625}+\mathcal{O}\left(h^{6}\right)=1.4222210^{-3} h^{5} y^{(5)}\left(t_{n}\right)+\mathcal{O}\left(h^{6}\right) .
\end{aligned}
$$

Then the order of HBM1 is $(3,3,3,3)$ and the error constant is $(0,(14 / 46875),(-1 / 360),(8 / 5625))$. Therefore, the HBM1 is given as follows:

$$
\begin{aligned}
& y_{n+1}=y_{n}+h y_{n}^{\prime}+\frac{h^{2}}{72}\left(9 f_{n}+25 f_{n+k}+2 f_{n+1}\right), \\
& y_{n+k}=y_{n}+\frac{2 h}{5} y_{n}^{\prime}+\frac{2 h^{2}}{1125}\left(27 f_{n}+20 f_{n+k}-2 f_{n+1}\right), \\
& y_{n+1}^{\prime}=y_{n}^{\prime}+\frac{h}{36}\left(3 f_{n}+25 f_{n+k}+8 f_{n+1}\right), \\
& y_{n+k}^{\prime}=y_{n}^{\prime}+\frac{h}{225}\left(39 f_{n}+55 f_{n+k}-4 f_{n+1}\right) .
\end{aligned}
$$

\section{Analysis of the Proposed Method}

In this section, we study the main properties of the proposed method such as consistency, stability, and convergence. Let us write system (28) in the form

$$
\begin{aligned}
y_{n+(2 / 5)} & =y_{n}+\frac{2 h}{5} y_{n}^{\prime}+\frac{2 h^{2}}{1125}\left(27 f_{n}+20 f_{n+(2 / 5)}-2 f_{n+1}\right), \\
y_{n+1} & =y_{n}+h y_{n}^{\prime}+\frac{h^{2}}{72}\left(9 f_{n}+25 f_{n+(2 / 5)}+2 f_{n+1}\right), \\
h y_{n+(2 / 5)}^{\prime} & =h y_{n}^{\prime}+\frac{h^{2}}{225}\left(39 f_{n}+55 f_{n+(2 / 5)}-4 f_{n+1}\right), \\
h y_{n+1}^{\prime} & =h y_{n}^{\prime}+\frac{h^{2}}{36}\left(3 f_{n}+25 f_{n+(2 / 5)}+8 f_{n+1}\right) .
\end{aligned}
$$


Then, we can rewrite system (29) in the matrix form as where

$$
A_{0} Y_{m}=A_{1} y_{m}+A_{2} F_{m}
$$

$$
\begin{aligned}
& Y_{m}=\left(\begin{array}{c}
y_{n+(2 / 5)} \\
y_{n+1} \\
h y_{n+(2 / 5)} \\
h y_{n+1},
\end{array}\right) \text {, } \\
& y_{m}=\left(\begin{array}{c}
y_{n-(2 / 5)} \\
y_{n} \\
h y_{n-(2 / 5)}^{\prime} \\
h y_{n}^{\prime}
\end{array}\right) \text {, } \\
& F_{m}=\left(\begin{array}{c}
h^{2} f_{n} \\
h^{2} f_{n+(2 / 5)} \\
h^{2} f_{n+1}
\end{array}\right) \text {, } \\
& A_{0}=\left(\begin{array}{llll}
1 & 0 & 0 & 0 \\
0 & 1 & 0 & 0 \\
0 & 0 & 1 & 0 \\
0 & 0 & 0 & 1
\end{array}\right), \\
& A_{1}=\left(\begin{array}{llll}
0 & 1 & 0 & \frac{2}{5} \\
0 & 1 & 0 & 1 \\
0 & 0 & 0 & 1 \\
0 & 0 & 0 & 1
\end{array}\right), \\
& A_{2}=\left(\begin{array}{ccc}
\frac{54}{1125} & \frac{40}{1125} & \frac{-4}{1125} \\
\frac{9}{72} & \frac{25}{72} & \frac{2}{72} \\
\frac{39}{225} & \frac{55}{225} & \frac{-4}{225} \\
\frac{3}{36} & \frac{25}{36} & \frac{8}{36}
\end{array}\right) .
\end{aligned}
$$


Following Fatunla's approach [29], the characteristic equation of HBM1 is

$$
\operatorname{det}\left(\mu A_{0}-A_{1}\right)=\operatorname{det}\left(\begin{array}{cccc}
\mu & -1 & 0 & -\frac{2}{5} \\
0 & \mu-1 & 0 & 1 \\
0 & 0 & \mu & -1 \\
0 & 0 & 0 & \mu-1
\end{array}\right)=\mu^{2}(\mu-1)^{2}=0 \text {, }
$$

which implies that $\mu_{1}=\mu_{2}=0$ and $\mu_{3}=\mu_{4}=1$. Then, the multiplicity of the nonzero roots of the characteristic equation is 2 which does not exceed the order of the differential equation. Hence, it is zero stable.

From Section 3, we see that the local truncation error of system (28) is

$$
\begin{aligned}
\mathscr{L}\left[y\left(t_{n}\right) ; h\right] & =\left(\mathscr{L}\left[y\left(t_{n+(2 / 5)}\right) ; h\right], \mathscr{L}\left[y\left(t_{n+1}\right) ; h\right], \mathscr{L}\left[y^{\prime}\left(t_{n+(2 / 5)}\right) ; h\right], \mathscr{L}\left[y^{\prime}\left(t_{n+1}\right) ; h\right]\right) \\
& =\sum_{i=0}^{\infty} \gamma_{i} h^{i} y^{(i)}\left(t_{n}\right)=\gamma_{5} h^{5} y^{(5)}\left(t_{n}\right)+\sum_{i=6}^{\infty} \gamma_{i} h^{i} y^{(i)}\left(t_{n}\right),
\end{aligned}
$$

where $\gamma_{0}=\gamma_{1}=\gamma_{2}=\gamma_{3}=\gamma_{4}=0$ and $\gamma_{5}=(0,(14 / 46875)$, (8/5625), (1/360) $)^{T}$.

Thus, system $(28)$ has order $(3,3,3,3)^{T}$. For simplicity, we write the order as 3 . Since the order of system (28) is $3>1$, then it is consistent. The consistency and the zero stability of system (28) imply that it is convergent $[11,13]$.

To find the region of absolute stability, we consider the following test problem $y^{\prime}=\lambda y$ where $\lambda<0$, then

$$
y^{\prime \prime}=f\left(t, y, y^{\prime}\right)=\lambda^{2} y .
$$

Substitute $f$ in the following matrix form:

$$
B_{0} Y_{n+1}=B_{1} Y_{n}+h B_{2} Y_{n+1}^{\prime}+h^{2}\left(C_{0} F_{n}+C_{1} F_{n+1}\right) \text {, }
$$

where

$$
\begin{aligned}
& Y_{n+1}=\left(\begin{array}{c}
y_{n+(2 / 5)} \\
y_{n+1}
\end{array}\right) \text {, } \\
& Y_{n}=\left(\begin{array}{c}
y_{n-(2 / 5)} \\
y_{n}
\end{array}\right) \text {, } \\
& Y_{n}^{\prime}=\left(\begin{array}{c}
y_{n-(2 / 5)}^{\prime} \\
y_{n}^{\prime}
\end{array}\right) \text {, } \\
& F_{n}=\left(\begin{array}{c}
f_{n-(2 / 5)} \\
f_{n}
\end{array}\right) \text {, } \\
& F_{n+1}=\left(\begin{array}{c}
f_{n+(2 / 5)} \\
f_{n+1}
\end{array}\right) \text {, } \\
& B_{0}=\left(\begin{array}{ll}
1 & 0 \\
0 & 1
\end{array}\right) \text {, } \\
& B_{1}=\left(\begin{array}{ll}
0 & 1 \\
0 & 1
\end{array}\right) \text {, }
\end{aligned}
$$




$$
\begin{aligned}
& B_{2}=\left(\begin{array}{ll}
0 & \frac{2}{5} \\
0 & 1
\end{array}\right), \\
& C_{0}=\left(\begin{array}{cc}
0 & \frac{54}{1125} \\
0 & \frac{9}{72}
\end{array}\right), \\
& C_{1}=\left(\begin{array}{cc}
\frac{40}{1125} & \frac{-4}{1125} \\
0 \frac{25}{72} & \frac{2}{72}
\end{array}\right),
\end{aligned}
$$

to get

$$
y^{\prime \prime}(t)=f\left(t, y, y^{\prime}\right), \quad t \geq 0
$$

$$
B_{0} Y_{n+1}=B_{1} Y_{n}+h B_{2} \lambda Y_{n}+h^{2}\left(C_{0} \lambda^{2} Y_{n}+C_{1} \lambda^{2} Y_{n+1}\right) .
$$

$$
y(0)=\hat{a},
$$

Let $S=\lambda h$, then

$$
Y_{n+1}=M(S) Y_{n},
$$

where $M(S)=\left(B_{0}-S^{2} C 1\right)^{-1}\left(B_{1}+S B_{2}+S^{2} C_{0}\right)$. The eigenvalue of $M(S)$ is

$$
\left(0, \frac{900+900 S+393 S^{2}+93 S^{3}+11 S^{4}}{900-57 S^{2}+2 S^{4}}\right) .
$$

Let $f: \mathbb{C} \longrightarrow \mathbb{C}$ by $f(S)=\left(900+900 S+393 S^{2}+93 S^{3}+\right.$ $\left.11 S^{4} / 900-57 S^{2}+2 S^{4}\right)$ where $S=\lambda h$. The region of absolute stability will be all $S \in \mathbb{C}$ such that $|f(S)|<1$. This region is given in Figure 2 and the interval of stability is $(-4.08611,0)$.

\section{Fuzzy Initial Value Problem}

$$
y^{\prime}(0)=\widehat{b} .
$$

Let the $\beta$-level of the solution $y(t), \widehat{a}, \widehat{b}$ and the function $f\left(t, y, y^{\prime}\right)$ be given by

$$
\begin{aligned}
y(t, \beta) & =\left[y_{1}(t, \beta), y_{2}(t, \beta)\right], \\
y^{\prime}(t, \beta) & =\left[y_{1}^{\prime}(t, \beta), y_{2}^{\prime}(t, \beta)\right], \\
y(0, \beta) & =\left[a_{1}, a_{2}\right], \\
y^{\prime}(0, \beta) & =\left[b_{1}, b_{2}\right], \\
f\left(t, y, y^{\prime}, \beta\right) & =\left[f_{1}\left(t, y(t, \beta), y^{\prime}(t, \beta)\right), f_{2}\left(t, y(t, \beta), y^{\prime}(t, \beta)\right)\right] .
\end{aligned}
$$

Following the technique described in previous sections, the fuzzy HBM1 is given by

In this section, we will apply HBM1 on the fuzzy initial value problem. We will present two new theorems. Consider the following fuzzy initial value problem

$$
\begin{gathered}
y_{1, n+1, \beta}=y_{1, n, \beta}+h y_{1, n, \beta}^{\prime}+\frac{h^{2}}{72}\left(9 f_{1, n, \beta}+25 f_{1, n+(2 / 5), \beta}+2 f_{1, n+1, \beta}\right), \\
y_{1, n+(2 / 5), \beta}=y_{1, n, \beta}+\frac{2 h}{5} y_{1, n, \beta}^{\prime}+\frac{2 h^{2}}{1125}\left(27 f_{1, n, \beta}+20 f_{1, n+(2 / 5), \beta}-2 f_{1, n+1, \beta}\right), \\
y_{1, n+1, \beta}^{\prime}=y_{1, n, \beta}^{\prime}+\frac{h}{36}\left(3 f_{1, n, \beta}+25 f_{1, n+(2 / 5), \beta}+8 f_{1, n+1, \beta}\right), \\
y_{1, n+(2 / 5), \beta}^{\prime}=y_{1, n, \beta}^{\prime}+\frac{h}{225}\left(39 f_{1, n, \beta}+55 f_{1, n+(2 / 5), \beta}-4 f_{1, n+1, \beta}\right), \\
y_{2, n+1, \beta}=y_{2, n, \beta}+h y_{2, n, \beta}^{\prime}+\frac{h^{2}}{72}\left(9 f_{2, n, \beta}+25 f_{2, n+(2 / 5), \beta}+2 f_{2, n+1, \beta}\right),
\end{gathered}
$$




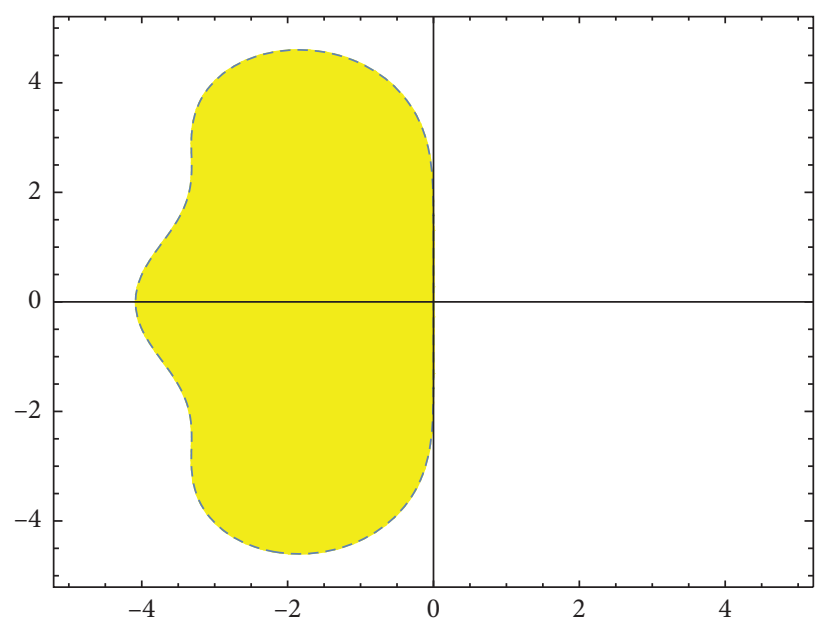

FIGURE 2: Region of absolute stability.

$$
\begin{aligned}
y_{2, n+(2 / 5), \beta} & =y_{2, n, \beta}+\frac{2 h}{5} y_{2, n, \beta}^{\prime}+\frac{2 h^{2}}{1125}\left(27 f_{2, n, \beta}+20 f_{2, n+(2 / 5), \beta}-2 f_{2, n+1, \beta}\right), \\
y_{2, n+1, \beta}^{\prime} & =y_{2, n, \beta}^{\prime}+\frac{h}{36}\left(3 f_{2, n, \beta}+25 f_{2, n+(2 / 5), \beta}+8 f_{2, n+1, \beta}\right), \\
y_{2, n+(2 / 5), \beta}^{\prime} & =y_{2, n, \beta}^{\prime}+\frac{h}{225}\left(39 f_{2, n, \beta}+55 f_{2, n+(2 / 5), \beta}-4 f_{2, n+1, \beta}\right),
\end{aligned}
$$

where

$$
\begin{aligned}
f_{1, n, \beta} & =\min \left\{f\left(t_{n}, w, w^{\prime}\right): w \in\left[y_{1, n, \beta}, y_{2, n, \beta}\right], w^{\prime} \in\left[y_{1, n, \beta}, y_{2, n, \beta}^{\prime}\right]\right\}, \\
f_{2, n, \beta} & =\max \left\{f\left(t_{n}, w, w^{\prime}\right): w \in\left[y_{1, n, \beta}, y_{2, n, \beta}\right], w^{\prime} \in\left[y_{1, n, \beta}, y_{2, n, \beta}^{\prime}\right]\right\}, \\
f_{1, n+(2 / 5), \beta} & =\min \left\{f\left(t_{n+(2 / 5)}, w, w^{\prime}\right): w \in\left[y_{1, n+(2 / 5), \beta}, y_{2, n+(2 / 5), \beta}\right], w^{\prime} \in\left[y_{1, n+(2 / 5), \beta}, y_{2, n+(2 / 5), \beta}\right]\right\}, \\
f_{2, n+(2 / 5), \beta} & =\max \left\{f\left(t_{n+(2 / 5)}, w, w^{\prime}\right): w \in\left[y_{1, n+(2 / 5), \beta}, y_{2, n+(2 / 5), \beta}\right], w^{\prime} \in\left[y_{1, n+(2 / 5), \beta}, y_{2, n+(2 / 5), \beta}\right]\right\}, \\
f_{1, n+1, \beta} & =\min \left\{f\left(t_{n+1}, w, w^{\prime}\right): w \in\left[y_{1, n+1, \beta}, y_{2, n+1, \beta}\right], w^{\prime} \in\left[y_{1, n+1, \beta}^{\prime}, y_{2, n+1, \beta}\right]\right\}, \\
f_{2, n+1, \beta} & =\max \left\{f\left(t_{n+1}, w, w^{\prime}\right): w \in\left[y_{1, n+1, \beta}, y_{2, n+1, \beta}\right], w^{\prime} \in\left[y_{1, n+1, \beta}, y_{2, n+1, \beta}\right]\right\} .
\end{aligned}
$$

Theorem 2. Let $f\left(t, y, y^{\prime}\right)$ be increasing function in $y$ and $y^{\prime}$. Then the following are true.

(i) If $y>0$ and $y^{\prime}>0$, then

$$
f_{1, n+j, \beta}=f\left(t_{n+j}, y_{1, n+j, \beta}, y_{1, n+j, \beta}^{\prime}\right), f_{2, n+j, \beta}=f\left(t_{n+j}, y_{2, n+j, \beta}, y_{2, n+j, \beta}^{\prime}\right), \quad \text { for } j=0, \frac{2}{5}, 1
$$

(ii) If $y>0$ and $y^{\prime}<0$, then

$$
f_{1, n+j, \beta}=f\left(t_{n+j}, y_{1, n+j, \beta}, y_{2, n+j, \beta}^{\prime}\right), f_{2, n+j, \beta}=f\left(t_{n+j}, y_{2, n+j, \beta}, y_{1, n+j, \beta}^{\prime}\right), \quad \text { for } j=0, \frac{2}{5}, 1 .
$$


(iii) If $y<0$ and $y^{\prime}<0$, then

$$
f_{1, n+j, \beta}=f\left(t_{n+j}, y_{2, n+j, \beta}, y_{2, n+j, \beta}^{\prime}\right), f_{2, n+j, \beta}=f\left(t_{n+j}, y_{1, n+j, \beta}, y_{1, n+j, \beta}^{\prime}\right), \quad \text { for } j=0, \frac{2}{5}, 1 .
$$

(iv) If $y<0$ and $y^{\prime}>0$, then

$$
f_{1, n+j, \beta}=f\left(t_{n+j}, y_{2, n+j, \beta}, y_{1, n+j, \beta}^{\prime}\right), f_{2, n+j, \beta}=f\left(t_{n+j}, y_{1, n+j, \beta}, y_{2, n+j, \beta}^{\prime}\right), \quad \text { for } j=0, \frac{2}{5}, 1
$$

The proof of the theorem follows straight forward. We can generate the functions $f_{i, n+j, \beta}$ for $i=1,2$ and $j=0,(2 / 5), 1$ for the decreasing case in similar way as in Theorem 2.

Theorem 3. Let $\widehat{c}=\left[c_{1}, c_{2}\right]$ be a fuzzy number, $a, b$ be a real number and $f(t, y)=a y^{\prime}+b y+\widehat{c}$. Then the fuzzy system of HBM1 becomes
(1) If $a \geq 0$ and $b \geq 0$, then

$$
\begin{aligned}
& Y_{n+1}=A_{1}^{-1} B_{1} y_{j, n, \beta}+C_{1} A_{1}^{-1} y_{j, n, \beta}+D_{1} A_{1}^{-1} c_{j}, \\
& \text { for } j=1 \text { and 2, where }
\end{aligned}
$$

$$
\begin{aligned}
& A_{1}=\left(\begin{array}{rrrr}
1-\frac{8 b h^{2}}{225} & \frac{4 b h^{2}}{1125} & -\frac{8 a h^{2}}{225} & \frac{4 a h^{2}}{1125} \\
-\frac{25 b h^{2}}{72} & 1-\frac{2 b h^{2}}{72} & -\frac{25 a h^{2}}{72} & -\frac{2 a h^{2}}{72} \\
-\frac{55 b h}{225} & \frac{4 b h}{225} & 1-\frac{55 a h}{225} & \frac{4 a h}{225} \\
-\frac{25 b h}{36} & -\frac{8 b h}{36} & -\frac{25 a h}{36} & 1-\frac{8 a h}{36}
\end{array}\right), \\
& B_{1}=\left(\begin{array}{c}
\frac{6 a h^{2}}{125}+\frac{2 h}{5} \\
\frac{9 a h^{2}}{72}+h \\
\frac{39 a h}{225}+1 \\
\frac{3 a h}{36}+1
\end{array}\right) \\
& C_{1}=\left(\begin{array}{c}
\frac{6 b h^{2}}{125}+1 \\
\frac{9 b h^{2}}{72}+1 \\
\frac{39 b h}{225} \\
\frac{3 b h}{36}
\end{array}\right)
\end{aligned}
$$




$$
\begin{aligned}
D_{1} & =\left(\begin{array}{c}
\frac{2 h^{2}}{25} \\
\frac{h^{2}}{2} \\
\frac{2 h}{5} \\
h
\end{array}\right), \\
Y_{n+1} & =\left(\begin{array}{c}
y_{j, n+(2 / 5), \beta} \\
y_{j, n+1, \beta} \\
y_{j, n+(2 / 5), \beta} \\
y_{j, n+1, \beta}
\end{array}\right) .
\end{aligned}
$$

(2) If $a \geq 0$ and $b<0$, then

where

$$
Y_{n+1}=A_{2}^{-1} B_{2}\left(\begin{array}{c}
y_{1, n, \beta} \\
y_{2, n, \beta}
\end{array}\right)+A_{2}^{-1} C_{2}\left(\begin{array}{c}
y_{1, n, \beta} \\
y_{2, n, \beta}
\end{array}\right)+A_{2}^{-1} D_{2}\left(\begin{array}{l}
c_{1} \\
c_{2}
\end{array}\right) \text {, }
$$

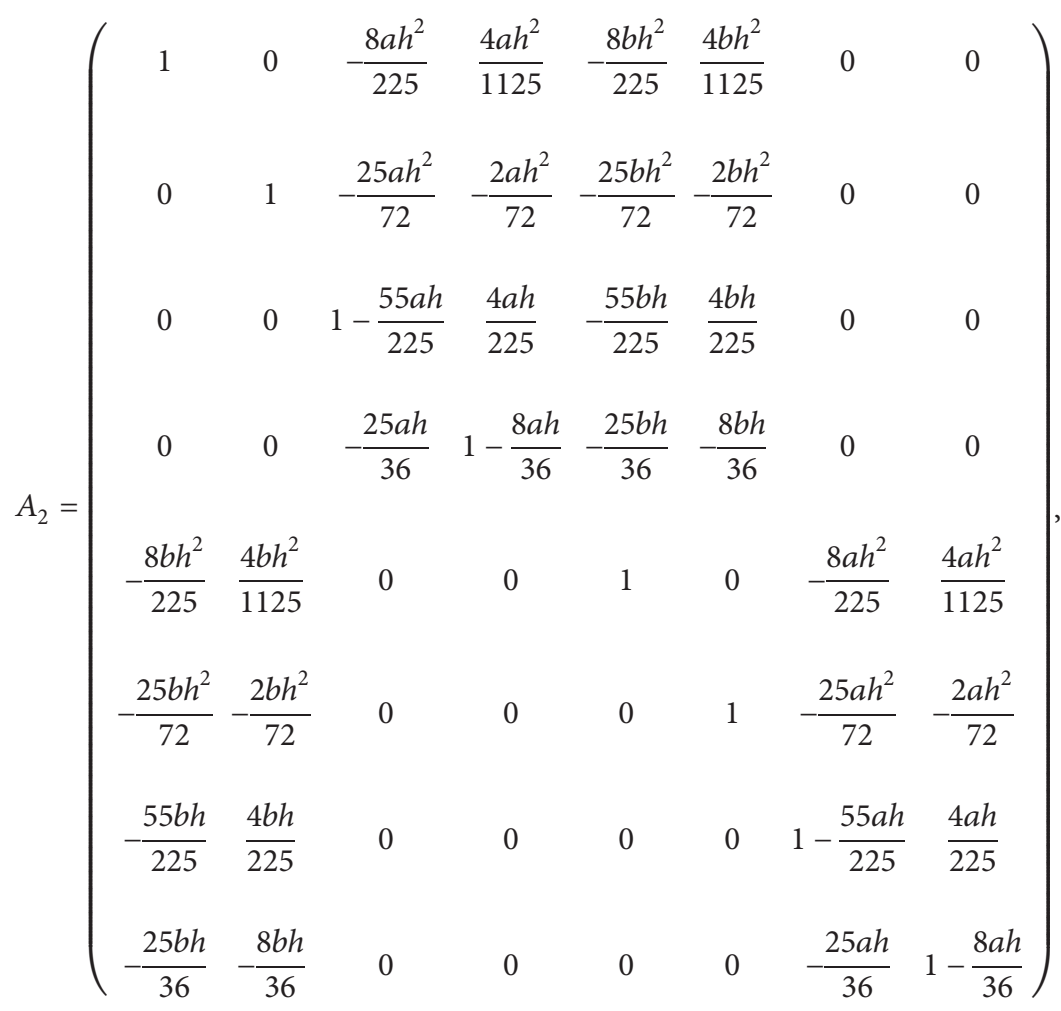




$$
\begin{aligned}
& B_{2}=\left(\begin{array}{cc}
\frac{6 a h^{2}}{125}+\frac{2 h}{5} & 0 \\
\frac{9 a h^{2}}{72}+h & 0 \\
\frac{39 a h}{225}+1 & 0 \\
\frac{3 a h}{36}+1 & 0 \\
0 & \frac{6 a h^{2}}{125}+\frac{2 h}{5} \\
0 & \frac{9 a h^{2}}{72}+h \\
0 & \frac{39 a h}{225}+1 \\
0 & \frac{3 a h}{36}+1
\end{array}\right), C_{2}=\left(\begin{array}{cc}
1 & \frac{6 b h^{2}}{125} \\
1 & \frac{9 b h^{2}}{72} \\
0 & \frac{39 b h}{225} \\
0 & \frac{3 b h}{36} \\
\frac{6 b h^{2}}{125} & 1 \\
\frac{9 b h^{2}}{72} & 1 \\
\frac{39 b h}{225} & 0 \\
\frac{3 b h}{36} & 0
\end{array}\right) \\
& D_{2}=\left(\begin{array}{cc}
\frac{2 h^{2}}{25} & 0 \\
\frac{h^{2}}{2} & 0 \\
\frac{2 h}{5} & 0 \\
h & 0 \\
0 & \frac{2 h^{2}}{25} \\
0 & \frac{h^{2}}{2} \\
0 & \frac{2 h}{5} \\
0 & h
\end{array}\right), Y_{n+1}=\left(\begin{array}{c}
y_{1, n+(2 / 5), \beta} \\
y_{1, n+1, \beta} \\
y_{1, n+(2 / 5), \beta} \\
y_{1, n+1, \beta} \\
y_{2, n+(2 / 5), \beta} \\
y_{2, n+1, \beta} \\
y_{2, n+(2 / 5), \beta} \\
y_{2, n+1, \beta}
\end{array}\right) . \\
& Y_{n+1}=A_{3}^{-1} B_{3}\left(\begin{array}{c}
y_{1, n, \beta}^{\prime} \\
y_{2, n, \beta}^{\prime}
\end{array}\right)+A_{3}^{-1} C_{3}\left(\begin{array}{l}
y_{1, n, \beta} \\
y_{2, n, \beta}
\end{array}\right)+A_{3}^{-1} D_{3}\left(\begin{array}{l}
c_{1} \\
c_{2}
\end{array}\right),
\end{aligned}
$$$$
\text { (3) If } a \leq 0 \text { and } b>0 \text {, then }
$$ 
where

$$
\begin{aligned}
& A_{3}=\left(\begin{array}{ccccccrc}
1-\frac{8 b h^{2}}{225} & \frac{4 b h^{2}}{1125} & 0 & 0 & 0 & 0 & -\frac{8 a h^{2}}{225} & \frac{4 a h^{2}}{1125} \\
-\frac{25 b h^{2}}{72} & 1-\frac{2 b h^{2}}{72} & 0 & 0 & 0 & 0 & -\frac{25 a h^{2}}{72} & -\frac{2 a h^{2}}{72} \\
-\frac{55 b h}{225} & \frac{4 b h}{225} & 1 & 0 & 0 & 0 & -\frac{55 a h}{225} & \frac{4 a h}{225} \\
-\frac{25 b h}{36} & -\frac{8 b h}{36} & 0 & 1 & 0 & 0 & -\frac{25 a h}{36} & -\frac{8 a h}{36} \\
0 & 0 & -\frac{8 a h^{2}}{225} & \frac{4 a h^{2}}{1125} & 1-\frac{8 b h^{2}}{225} & \frac{4 b h^{2}}{1125} & 0 & 0 \\
0 & 0 & -\frac{25 a h^{2}}{72} & -\frac{2 a h^{2}}{72} & -\frac{25 b h^{2}}{72} & 1-\frac{2 b h^{2}}{72} & 0 & 0 \\
0 & 0 & -\frac{55 a h}{225} & \frac{4 a h}{225} & -\frac{55 b h}{225} & \frac{4 b h}{225} & 1 & 0 \\
0 & -\frac{25 a h}{36} & -\frac{8 a h}{36} & -\frac{25 b h}{36} & -\frac{8 b h}{36} & 0 & 1
\end{array}\right), \\
& B_{3}=\left(\begin{array}{cc}
\frac{2 h}{5} & \frac{6 a h^{2}}{125} \\
h & \frac{9 a h^{2}}{72} \\
1 & \frac{39 a h}{225} \\
1 & \frac{3 a h}{36} \\
\frac{6 a h^{2}}{125} & \frac{2 h}{5} \\
\frac{9 a h^{2}}{72} & h \\
\frac{39 a h}{225} & 1 \\
\frac{3 a h}{36} & 1
\end{array}\right), C_{3}=\left(\begin{array}{cc}
\frac{6 b h^{2}}{125}+1 & 0 \\
\frac{9 b h^{2}}{72}+1 & 0 \\
\frac{39 b h}{225} & 0 \\
\frac{3 b h}{36} & 0 \\
0 & \frac{6 b h^{2}}{125}+1 \\
0 & \frac{9 b h^{2}}{72} \\
0 & \frac{39 b h}{225} \\
0 & \frac{3 b h}{36}
\end{array}\right), \\
& D_{3}=\left(\begin{array}{cc}
\frac{2 h^{2}}{25} & 0 \\
\frac{h^{2}}{2} & 0 \\
\frac{2 h}{5} & 0 \\
h & 0 \\
0 & \frac{2 h^{2}}{25} \\
0 & \frac{h^{2}}{2} \\
0 & \frac{2 h}{5} \\
0 & h
\end{array}\right), Y_{n+1}=\left(\begin{array}{c}
y_{1, n+(2 / 5), \beta} \\
y_{1, n+1, \beta} \\
y_{1, n+(2 / 5), \beta} \\
y_{1, n+1, \beta} \\
y_{2, n+(2 / 5), \beta} \\
y_{2, n+1, \beta} \\
y_{2, n+(2 / 5), \beta} \\
y_{2, n+1, \beta}
\end{array}\right) .
\end{aligned}
$$


(4) If $a \leq 0$ and $b<0$, then

where

$$
Y_{n+1}=A_{4}^{-1} B_{4}\left(\begin{array}{c}
y_{1, n, \beta}^{\prime} \\
y_{2, n, \beta}
\end{array}\right)+A_{4}^{-1} C_{4}\left(\begin{array}{l}
y_{1, n, \beta} \\
y_{2, n, \beta}
\end{array}\right)+A_{4}^{-1} D_{4}\left(\begin{array}{l}
c_{1} \\
c_{2}
\end{array}\right)
$$

$$
\begin{aligned}
& A_{4}=\left(\begin{array}{cccccccc}
1 & 0 & 0 & 0 & -\frac{8 b h^{2}}{225} & \frac{4 b h^{2}}{1125} & -\frac{8 a h^{2}}{225} & \frac{4 a h^{2}}{1125} \\
0 & 1 & 0 & 0 & -\frac{25 b h^{2}}{72} & -\frac{2 b h^{2}}{72} & -\frac{25 a h^{2}}{72} & -\frac{2 a h^{2}}{72} \\
0 & 0 & 1 & 0 & -\frac{55 b h}{225} & \frac{4 b h}{225} & -\frac{55 a h}{225} & \frac{4 a h}{225} \\
0 & 0 & 0 & 1 & -\frac{25 b h}{36} & -\frac{8 b h}{36} & -\frac{25 a h}{36} & -\frac{8 a h}{36} \\
-\frac{8 b h^{2}}{225} & \frac{4 b h^{2}}{1125} & -\frac{8 a h^{2}}{225} & \frac{4 a h^{2}}{1125} & 1 & 0 & 0 & 0 \\
-\frac{25 b h^{2}}{72} & -\frac{2 b h^{2}}{72} & -\frac{25 a h^{2}}{72} & -\frac{2 a h^{2}}{72} & 0 & 1 & 0 & 0 \\
-\frac{25 b h}{36} & -\frac{8 b h}{36} & -\frac{25 a h}{36} & -\frac{8 a h}{36} & 0 & 0 & 0 & 1
\end{array}\right) \\
& B_{4}=\left(\begin{array}{cc}
\frac{2 h}{5} & \frac{6 a h^{2}}{125} \\
h & \frac{9 a h^{2}}{72} \\
1 & \frac{39 a h}{225} \\
1 & \frac{3 a h}{36} \\
\frac{6 a h^{2}}{125} & \frac{2 h}{5} \\
\frac{9 a h^{2}}{72} & h \\
\frac{39 a h}{225} & 1 \\
\frac{3 a h}{36} & 1
\end{array}\right), C_{4}=\left(\begin{array}{cc}
1 & \frac{6 b h^{2}}{125} \\
0 & \frac{39 b h}{225} \\
0 & \frac{3 b h}{36} \\
\frac{6 b h^{2}}{125} & 1 \\
\frac{9 b h^{2}}{72} & 1 \\
\frac{39 b h}{225} & 0 \\
\frac{3 b h}{36} & 0
\end{array}\right)
\end{aligned}
$$




$$
D_{4}=\left(\begin{array}{cc}
\frac{2 h^{2}}{25} & 0 \\
\frac{h^{2}}{2} & 0 \\
\frac{2 h}{5} & 0 \\
h & 0 \\
0 & \frac{2 h^{2}}{25} \\
0 & \frac{h^{2}}{2} \\
0 & \frac{2 h}{5} \\
0 & h
\end{array}\right), Y_{n+1}=\left(\begin{array}{c}
y_{1, n+(2 / 5), \beta} \\
y_{1, n+1, \beta} \\
y_{1, n+(2 / 5), \beta} \\
y_{1, n+1, \beta} \\
y_{2, n+(2 / 5), \beta} \\
y_{2, n+1, \beta} \\
y_{2, n+(2 / 5), \beta} \\
y_{2, n+1, \beta}
\end{array}\right) .
$$

Proof. (1) Let $a \geq 0$ and $b \geq 0$. Then,

$$
f\left(t_{n+j}, y_{i, n+j, \beta}, y_{i, n+j, \beta}^{\prime}\right)=c_{i}+b y_{i, n+j, \beta}+a y_{i, n+j, \beta}^{\prime},
$$

$$
\begin{aligned}
y_{1, n+1, \beta}= & y_{1, n, \beta}+h y_{1, n, \beta}^{\prime}+\frac{h^{2}}{72}\left(9\left(c_{1}+b y_{1, n, \beta}+a y_{1, n, \beta}^{\prime}\right)+25\left(c_{1}+b y_{1, n+(2 / 5), \beta}+a y_{1, n+(2 / 5), \beta}^{\prime}\right)+2\left(c_{1}+b y_{1, n+1, \beta}+a y_{1, n+1, \beta}^{\prime}\right)\right) \\
y_{1, n+(2 / 5), \beta}= & y_{1, n, \beta}+\frac{2 h}{5} y_{1, n, \beta}^{\prime}+\frac{2 h^{2}}{1125}\left(27\left(c_{1}+b y_{1, n, \beta}+a y_{1, n, \beta}^{\prime}\right)+20\left(c_{1}+b y_{1, n+(2 / 5), \beta}+a y_{1, n+(2 / 5), \beta}^{\prime}\right)\right. \\
& \left.-2\left(c_{1}+b y_{1, n+1, \beta}+a y_{1, n+1, \beta}^{\prime}\right)\right) \\
y_{1, n+1, \beta}^{\prime}= & y_{1, n, \beta}^{\prime}+\frac{h}{36}\left(3\left(c_{1}+b y_{1, n, \beta}+a y_{1, n, \beta}^{\prime}\right)+25\left(c_{1}+b y_{1, n+(2 / 5), \beta}+a y_{1, n+(2 / 5), \beta}^{\prime}\right)\right. \\
& \left.+8\left(c_{1}+b y_{1, n+1, \beta}+a y_{1, n+1, \beta}^{\prime}\right)\right) \\
y_{1, n+(2 / 5), \beta}^{\prime}= & y_{1, n, \beta}^{\prime}+\frac{h}{225}\left(39\left(c_{1}+b y_{1, n, \beta}+a y_{1, n, \beta}^{\prime}\right)+55\left(c_{1}+b y_{1, n+(2 / 5), \beta}+a y_{1, n+(2 / 5), \beta}^{\prime}\right)\right. \\
& \left.-4\left(c_{1}+b y_{1, n+1, \beta}+a y_{1, n+1, \beta}^{\prime}\right)\right) \\
& \left.+2\left(c_{2}+b y_{2, n+1, \beta}+a y_{2, n+1, \beta}^{\prime}\right)\right)
\end{aligned}
$$




$$
\begin{aligned}
y_{2, n+(2 / 5), \beta}= & y_{2, n, \beta}+\frac{2 h}{5} y_{2, n, \beta}^{\prime}+\frac{2 h^{2}}{1125}\left(27\left(c_{2}+b y_{2, n, \beta}+a y_{2, n, \beta}^{\prime}\right)+20\left(c_{2}+b y_{2, n+(2 / 5), \beta}+a y_{2, n+(2 / 5), \beta}^{\prime}\right)\right. \\
& \left.-2\left(c_{2}+b y_{2, n+1, \beta}+a y_{2, n+1, \beta}^{\prime}\right)\right), \\
y_{2, n+1, \beta}^{\prime}= & y_{2, n, \beta}^{\prime}+\frac{h}{36}\left(3\left(c_{2}+b y_{2, n, \beta}+a y_{2, n, \beta}^{\prime}\right)+25\left(c_{2}+b y_{2, n+(2 / 5), \beta}+a y_{2, n+(2 / 5), \beta}^{\prime}\right)\right. \\
& \left.+8\left(c_{2}+b y_{2, n+1, \beta}+a y_{2, n+1, \beta}^{\prime}\right)\right), \\
\prime & y_{2, n, \beta}^{\prime}+\frac{h}{225}\left(39\left(c_{2}+b y_{2, n, \beta}+a y_{2, n, \beta}^{\prime}\right)+55\left(c_{2}+b y_{2, n+(2 / 5), \beta}+a y_{2, n+(2 / 5), \beta}^{\prime}\right)\right. \\
& \left.-4\left(c_{2}+b y_{2, n+1, \beta}+a y_{2, n+1, \beta}^{\prime}\right)\right) .
\end{aligned}
$$

We can rewrite the last system in the matrix form as

$$
A_{1} Y_{1}=B_{1} y_{1, n, \beta}^{\prime}+C_{1} y_{1, n, \beta}+D_{1} c_{1}, A_{1} Y_{2}=B_{1} y_{2, n, \beta}^{\prime}+C_{1} y_{2, n, \beta}+D_{1} c_{2}
$$

where

$$
A_{1}=\left(\begin{array}{cccc}
1-\frac{8 b h^{2}}{225} & \frac{4 b h^{2}}{1125} & -\frac{8 a h^{2}}{225} & \frac{4 a h^{2}}{1125} \\
-\frac{25 b h^{2}}{72} & 1-\frac{2 b h^{2}}{72} & -\frac{25 a h^{2}}{72} & -\frac{2 a h^{2}}{72} \\
-\frac{55 b h}{225} & \frac{4 b h}{225} & 1-\frac{55 a h}{225} & \frac{4 a h}{225} \\
-\frac{25 b h}{36} & -\frac{8 b h}{36} & -\frac{25 a h}{36} & 1-\frac{8 a h}{36}
\end{array}\right), Y_{1}=\left(\begin{array}{c}
y_{1, n+(2 / 5), \beta} \\
y_{1, n+1, \beta} \\
y_{1, n+(2 / 5), \beta} \\
y_{1, n+1, \beta}
\end{array}\right), Y_{2}=\left(\begin{array}{c}
y_{2, n+(2 / 5), \beta} \\
y_{2, n+1, \beta} \\
y_{2, n+(2 / 5), \beta} \\
y_{2, n+1, \beta}
\end{array}\right),
$$

Since

$$
\operatorname{det}\left(A_{1}\right)=\frac{43740000 a^{2} h^{2}+15309000 a b h^{3}-306180000 a h+1458000 b^{2} h^{4}-41553000 b h^{2}+656100000}{656100000} \neq 0
$$


then $A_{1}^{-1}$ exist. Thus,

where

$$
\left(\begin{array}{c}
y_{1, n+(2 / 5), \beta} \\
y_{1, n+1, \beta} \\
y_{1, n+(2 / 5), \beta} \\
y_{1, n+1, \beta}
\end{array}\right)=E_{1} y_{1, n, \beta}{ }^{\prime}+E_{2} y_{1, n, \beta}+E_{3} c_{1},
$$

$$
\begin{aligned}
& E_{1}=A_{1}^{-1} B_{1}=\left(\begin{array}{c}
\frac{2 h\left(h\left(a\left(3 a b h^{3}+16\left(a^{2}+b\right) h^{2}-1200\right)-165 b h\right)+4500\right)}{25\left(h\left(60 h a^{2}+21\left(b h^{2}-20\right) a+b h\left(2 b h^{2}-57\right)\right)+900\right)} \\
\frac{h\left(h\left(186 b h+a\left(-3 a b h^{3}-5\left(a^{2}+b\right) h^{2}+60\right)\right)+1800\right)}{2 h\left(60 h a^{2}+21\left(b h^{2}-20\right) a+b h\left(2 b h^{2}-57\right)\right)+1800} \\
\frac{22500-h\left(4 h\left(8 b h^{2}+225\right) a^{2}+3\left(b\left(2 b h^{2}+135\right) h^{2}+500\right) a+3 b h\left(24 b h^{2}-125\right)\right)}{25\left(h\left(60 h a^{2}+21\left(b h^{2}-20\right) a+b h\left(2 b h^{2}-57\right)\right)+900\right)} \\
\frac{h\left(5 h\left(b h^{2}+36\right) a^{2}+3\left(b h^{2}+16\right)\left(b h^{2}+20\right) a+3 b h\left(9 b h^{2}+262\right)\right)+1800}{2 h\left(60 h a^{2}+21\left(b h^{2}-20\right) a+b h\left(2 b h^{2}-57\right)\right)+1800}
\end{array}\right), \\
& E_{2}=A_{1}^{-1} C_{1}=\left(\begin{array}{c}
\frac{6 a b^{2} h^{5}+8 b\left(4 a^{2}-5 b\right) h^{4}-75 a b h^{3}+375\left(4 a^{2}+b\right) h^{2}-10500 a h+22500}{25\left(h\left(60 h a^{2}+21\left(b h^{2}-20\right) a+b h\left(2 b h^{2}-57\right)\right)+900\right)} \\
\frac{h\left(-5 h\left(b h^{2}-24\right) a^{2}-3\left(b\left(b h^{2}+26\right) h^{2}+280\right) a+2 b h\left(11 b h^{2}+393\right)\right)+1800}{2 h\left(60 h a^{2}+21\left(b h^{2}-20\right) a+b h\left(2 b h^{2}-57\right)\right)+1800} \\
-\frac{2 b h\left(b h^{2}+75\right)\left(3 b h^{2}+16 a h-60\right)}{25\left(h\left(60 h a^{2}+21\left(b h^{2}-20\right) a+b h\left(2 b h^{2}-57\right)\right)+900\right)} \\
\frac{b h\left(b h^{2}+12\right)\left(3 b h^{2}+5 a h+150\right)}{2 h\left(60 h a^{2}+21\left(b h^{2}-20\right) a+b h\left(2 b h^{2}-57\right)\right)+1800}
\end{array}\right) \\
& E_{3}=A_{1}^{-1} D_{1}=\left(\begin{array}{c}
\frac{2 h^{2}(a h-15)\left(3 b h^{2}+16 a h-60\right)}{25\left(h\left(60 h a^{2}+21\left(b h^{2}-20\right) a+b h\left(2 b h^{2}-57\right)\right)+900\right)} \\
-\frac{h^{2}(a h-6)\left(3 b h^{2}+5 a h+150\right)}{2 h\left(60 h a^{2}+21\left(b h^{2}-20\right) a+b h\left(2 b h^{2}-57\right)\right)+1800} \\
-\frac{2 h\left(b h^{2}+75\right)\left(3 b h^{2}+16 a h-60\right)}{25\left(h\left(60 h a^{2}+21\left(b h^{2}-20\right) a+b h\left(2 b h^{2}-57\right)\right)+900\right)} \\
\frac{h\left(b h^{2}+12\right)\left(3 b h^{2}+5 a h+150\right)}{2 h\left(60 h a^{2}+21\left(b h^{2}-20\right) a+b h\left(2 b h^{2}-57\right)\right)+1800}
\end{array}\right) .
\end{aligned}
$$

Using similar argument, we can prove (2), (13), and (14). 
We end Section 5 by the following algorithm to solve problem (40)-(42).

(1) Let $y_{1,0, \beta}=a_{1}, y_{2,0, \beta}=a_{2}, y_{1,0, \beta}^{\prime}=b_{1}, y_{2,0, \beta}^{\prime}=b_{2}$, and $\varepsilon>0$

(2) For $n=1,2, \ldots$, do step 3 and 4

(3) Solve the fuzzy HBM1 to find $y_{1, n, \beta}, y_{2, n, \beta}, y_{1, n, \beta}, y_{2, n, \beta}$

(4) If $\left\|\left(y_{1, n, \beta}, y_{2, n, \beta}\right)-\left(y_{1, n-1, \beta}, y_{2, n-1, \beta}\right)\right\|<\varepsilon$ or $n>N$ max, go to step 5 , else $n=n+1$, go to step 3

(5) Stop

(6) If $n \leq N \max$, the approximate solution is $\left(y_{1, n, \beta}, y_{2, n, \beta}\right)$, else the method is divergent

\section{Numerical Examples}

In this section, we discuss four examples. Two are linear fuzzy initial value problems while the others are nonlinear.
Example 3. Consider the second-order fuzzy linear initial value problem

$$
y^{\prime \prime}=-y(x), y(0)=0, y^{\prime}(0)=\widehat{\gamma}_{\beta},
$$

where $\widehat{\gamma}_{\beta}=[0.9+0.1 \beta, 1.1-0.1 \beta]$ and $h=0.1$. Let $y(x)=$ $\left[y_{1}(x, \beta), y_{2}(x, \beta)\right]$ be a fuzzy solution and $y^{\prime}(x)=\left[y_{1}^{\prime}(x, \beta), y_{2}^{\prime}(x, \beta)\right]$.

By implementing the $\beta$-level sets, the problem will be

$$
\begin{aligned}
{\left[y_{1}^{\prime \prime}(x, \beta), y_{2}^{\prime \prime}(x, \beta)\right] } & =\left[-y_{2}(x, \beta),-y_{1}(x, \beta)\right], \\
{\left[y_{1}(0, \beta), y_{2}(0, \beta)\right] } & =\left[y_{1}^{\prime}(0, \beta), y_{2}^{\prime}(0, \beta)\right]=[\beta, 2-\beta] .
\end{aligned}
$$

Using HBM1, we get

$$
\begin{aligned}
y_{1, n+1, \beta} & =y_{1, n, \beta}+h y_{1, n, \beta}-\frac{h^{2}}{72}\left(9 y_{2, n, \beta}+25 y_{2, n+(2 / 5), \beta}+2 y_{2, n+1, \beta}\right), \\
y_{1, n+(2 / 5), \beta} & =y_{1, n, \beta}+\frac{2 h}{5} y_{1, n, \beta}^{\prime}-\frac{2 h^{2}}{1125}\left(27 y_{2, n, \beta}+20 y_{2, n+(2 / 5), \beta}-2 y_{2, n+1, \beta}\right), \\
y_{1, n+1, \beta} & =y_{1, n, \beta}-\frac{h}{36}\left(3 y_{2, n, \beta}+25 y_{2, n+(2 / 5), \beta}+8 y_{2, n+1, \beta}\right), \\
y_{1, n+(2 / 5), \beta} & =y_{1, n, \beta}-\frac{h}{225}\left(39 y_{2, n, \beta}+55 y_{2, n+(2 / 5), \beta}-4 y_{2, n+1, \beta}\right), \\
y_{2, n+1, \beta} & =y_{2, n, \beta}+h y_{2, n, \beta}^{\prime}-\frac{h^{2}}{72}\left(9 y_{1, n, \beta}+25 y_{1, n+(2 / 5), \beta}+2 y_{1, n+1, \beta}\right), \\
y_{2, n+(2 / 5), \beta} & =y_{2, n, \beta}+\frac{2 h}{5} y_{2, n, \beta}^{\prime}-\frac{2 h^{2}}{1125}\left(27 y_{1, n, \beta}+20 y_{1, n+(2 / 5), \beta}-2 y_{1, n+1, \beta}\right), \\
y_{2, n+1, \beta} & =y_{2, n, \beta}-\frac{h}{36}\left(3 y_{1, n, \beta}+25 y_{1, n+(2 / 5), \beta}+8 y_{1, n+1, \beta}\right), \\
y_{2, n+(2 / 5), \beta} & =y_{2, n, \beta}^{\prime}-\frac{h}{225}\left(39 y_{1, n, \beta}+55 y_{1, n+(2 / 5), \beta}-4 y_{1, n+1, \beta}\right) .
\end{aligned}
$$

We can rewrite the above system in the matrix form as

$$
Y_{m}=A^{-1} B y_{m}+A^{-1} C y_{m}^{\prime}
$$

where 


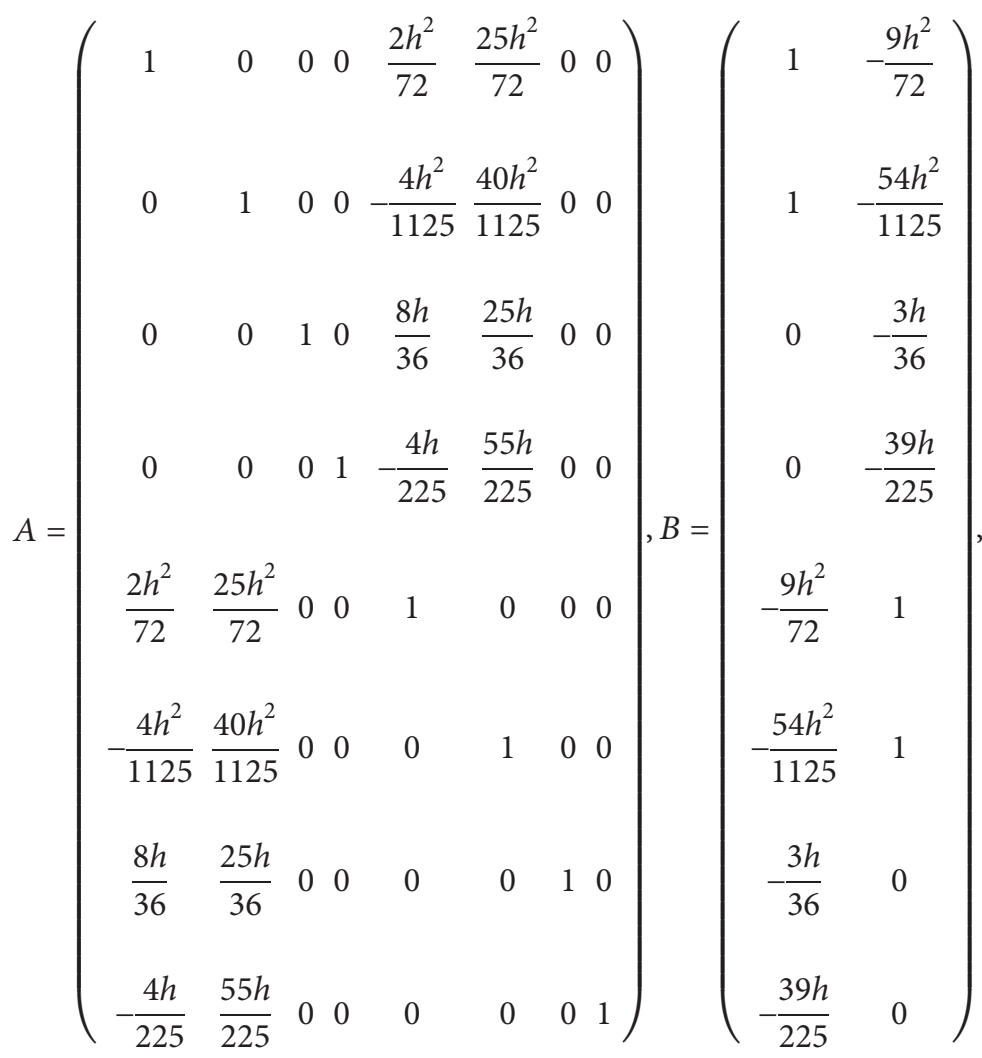

$$
\begin{aligned}
& C=\left(\begin{array}{cc}
h & 0 \\
\frac{2 h}{5} & 0 \\
1 & 0 \\
1 & 0 \\
0 & h \\
0 & \frac{2 h}{5} \\
0 & 1 \\
0 & 1
\end{array}\right), Y_{m}=\left(\begin{array}{c}
y_{1, n+1} \\
y_{1, K+n} \\
y_{1, n+1} \\
y_{1, k+n} \\
y_{2, n+1} \\
y_{2, K+n} \\
y_{2, n+1}^{\prime} \\
y_{2, k+n}
\end{array}\right), y_{m}=\left(\begin{array}{l}
y_{1, n} \\
y_{2, n}
\end{array}\right), y_{m}^{\prime}=\left(\begin{array}{c}
y_{1, n}^{\prime} \\
y_{2, n}^{\prime}
\end{array}\right) .
\end{aligned}
$$

The error in the approximation of $y_{1, n}$ and $y_{2, n}$ for $\beta=$ $0,0.25,0.5,0.75,1$ is given in Tables 1 and 2 , respectively, where the exact solution is given by

$$
\begin{aligned}
& {[(0.1 \beta-0.1) \sinh (x)+\sin (x),(0.1-0.1 \beta) \sinh (x)} \\
& \quad+\sin (x)] .
\end{aligned}
$$

The approximate solutions of $y_{1}$ and $y_{2}$ for $\beta=0,0.25,0.5,0.75,1$ are given in Figures 3 and 4 , respectively. Also, in Figure 5, the approximate solutions for $y_{1}$ and $y_{2}$ become close to the crisp solution as $\beta$ approaches to 1 .

Example 4. Consider the following nonlinear second-order fuzzy initial value problem

$$
y^{\prime \prime}(x)=-\left(y^{\prime}(x)\right)^{2}, y(0)=\widehat{\gamma}_{\beta}, y^{\prime}(0)=\widehat{\theta}_{\beta}, \quad 0 \leq x \leq 0.1,
$$


TABLE 1: The absolute error in approximating $y_{1, n}$ for $h=0.1$.

\begin{tabular}{|c|c|c|c|c|c|}
\hline & & & $y_{1, n}$ & & \\
\hline$x$ & & & $\beta$ & & \\
\hline$\lambda$ & 0 & 0.25 & 0.5 & 0.75 & 1 \\
\hline 0 & 0 & 0 & 0 & 0 & 0 \\
\hline 0.1 & $4.50043 \times 10^{-12}$ & $4.39764 \times 10^{-12}$ & $4.29483 \times 10^{-12}$ & $4.19204 \times 10^{-12}$ & $4.08924 \times 10^{-12}$ \\
\hline 0.2 & $2.49082 \times 10^{-8}$ & $2.56047 \times 10^{-8}$ & $2.63012 \times 10^{-8}$ & $2.69978 \times 10^{-8}$ & $2.76943 \times 10^{-8}$ \\
\hline 0.3 & $7.40714 \times 10^{-8}$ & $7.6176 \times 10^{-8}$ & $7.82805 \times 10^{-8}$ & $8.03851 \times 10^{-8}$ & $8.24896 \times 10^{-8}$ \\
\hline 0.4 & $1.46254 \times 10^{-7}$ & $1.50506 \times 10^{-7}$ & $1.54759 \times 10^{-7}$ & $1.59012 \times 10^{-7}$ & $1.63264 \times 10^{-7}$ \\
\hline 0.5 & $2.39628 \times 10^{-7}$ & $2.46812 \times 10^{-7}$ & $2.53997 \times 10^{-7}$ & $2.61182 \times 10^{-7}$ & $2.68366 \times 10^{-7}$ \\
\hline 0.6 & $3.51805 \times 10^{-7}$ & $3.62763 \times 10^{-7}$ & $3.73722 \times 10^{-7}$ & $3.8468 \times 10^{-7}$ & $3.95639 \times 10^{-7}$ \\
\hline 0.7 & $4.79859 \times 10^{-7}$ & $4.95509 \times 10^{-7}$ & $5.11159 \times 10^{-7}$ & $5.26808 \times 10^{-7}$ & $5.42458 \times 10^{-7}$ \\
\hline 0.8 & $6.20368 \times 10^{-7}$ & $6.41718 \times 10^{-7}$ & $6.63068 \times 10^{-7}$ & $6.84418 \times 10^{-7}$ & $7.05768 \times 10^{-7}$ \\
\hline 0.9 & $7.6945 \times 10^{-7}$ & $7.9762 \times 10^{-7}$ & $8.2579 \times 10^{-7}$ & $8.5396 \times 10^{-7}$ & $8.82131 \times 10^{-7}$ \\
\hline 1 & $9.22809 \times 10^{-7}$ & $9.59051 \times 10^{-7}$ & $9.95292 \times 10^{-7}$ & $1.03153 \times 10^{-6}$ & $1.06777 \times 10^{-6}$ \\
\hline
\end{tabular}

TABLE 2: The absolute error in approximating $y_{2, n}$ for $h=0.1$.

\begin{tabular}{|c|c|c|c|c|c|}
\hline & & & $y_{2, n}$ & & \\
\hline$x$ & & & $\beta$ & & \\
\hline$x$ & 0 & 0.25 & 0.5 & 0.75 & 1 \\
\hline 0 & 0 & 0 & 0 & 0 & 0 \\
\hline 0.1 & $3.67807 \times 10^{-12}$ & $3.78086 \times 10^{-12}$ & $3.88367 \times 10^{-12}$ & $3.98646 \times 10^{-12}$ & $4.08926 \times 10^{-12}$ \\
\hline 0.2 & $3.04804 \times 10^{-8}$ & $2.97839 \times 10^{-8}$ & $2.90874 \times 10^{-8}$ & $2.83908 \times 10^{-8}$ & $2.76943 \times 10^{-8}$ \\
\hline 0.3 & $9.09078 \times 10^{-8}$ & $8.88032 \times 10^{-8}$ & $8.66987 \times 10^{-8}$ & $8.45941 \times 10^{-8}$ & $8.24896 \times 10^{-8}$ \\
\hline 0.4 & $1.80275 \times 10^{-7}$ & $1.76023 \times 10^{-7}$ & $1.7177 \times 10^{-7}$ & $1.67517 \times 10^{-7}$ & $1.63264 \times 10^{-7}$ \\
\hline 0.5 & $2.97104 \times 10^{-7}$ & $2.8992 \times 10^{-7}$ & $2.82735 \times 10^{-7}$ & $2.75551 \times 10^{-7}$ & $2.68366 \times 10^{-7}$ \\
\hline 0.6 & $4.39473 \times 10^{-7}$ & $4.28515 \times 10^{-7}$ & $4.17556 \times 10^{-7}$ & $4.06598 \times 10^{-7}$ & $3.95639 \times 10^{-7}$ \\
\hline 0.7 & $6.05057 \times 10^{-7}$ & $5.89408 \times 10^{-7}$ & $5.73758 \times 10^{-7}$ & $5.58108 \times 10^{-7}$ & $5.42458 \times 10^{-7}$ \\
\hline 0.8 & $7.91169 \times 10^{-7}$ & $7.69819 \times 10^{-7}$ & $7.48469 \times 10^{-7}$ & $7.27119 \times 10^{-7}$ & $7.05768 \times 10^{-7}$ \\
\hline 0.9 & $9.94812 \times 10^{-7}$ & $9.66642 \times 10^{-7}$ & $9.38471 \times 10^{-7}$ & $9.10301 \times 10^{-7}$ & $8.82131 \times 10^{-7}$ \\
\hline 1 & $1.21274 \times 10^{-6}$ & $1.1765 \times 10^{-6}$ & $1.14026 \times 10^{-6}$ & $1.10402 \times 10^{-6}$ & $1.06777 \times 10^{-6}$ \\
\hline
\end{tabular}

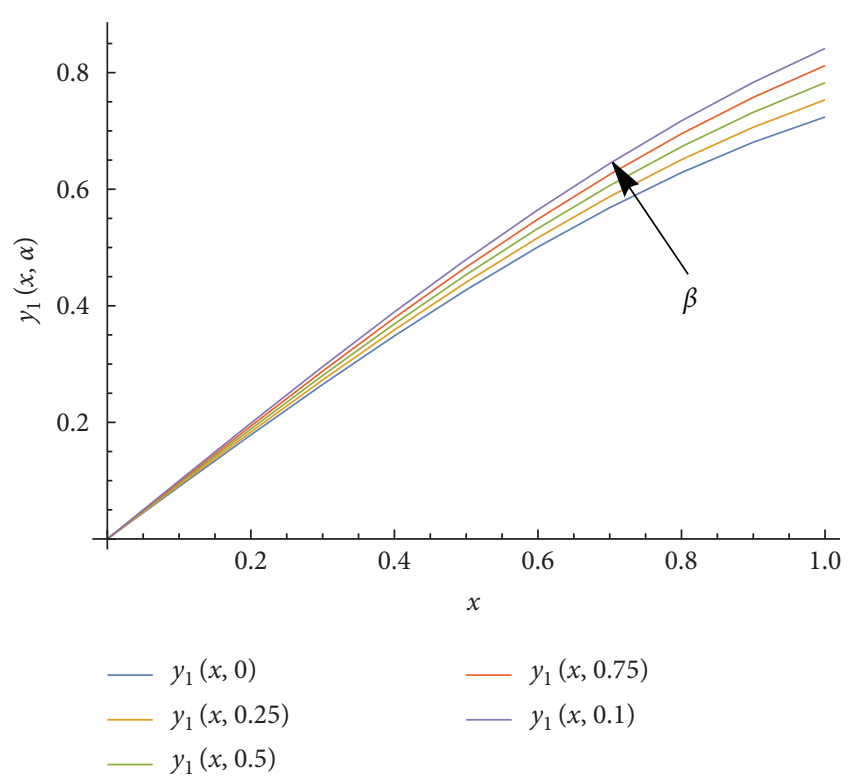

Figure 3: The approximate solution $y_{1}$ for $\beta=0,0.25,0.5,0.75,1$.

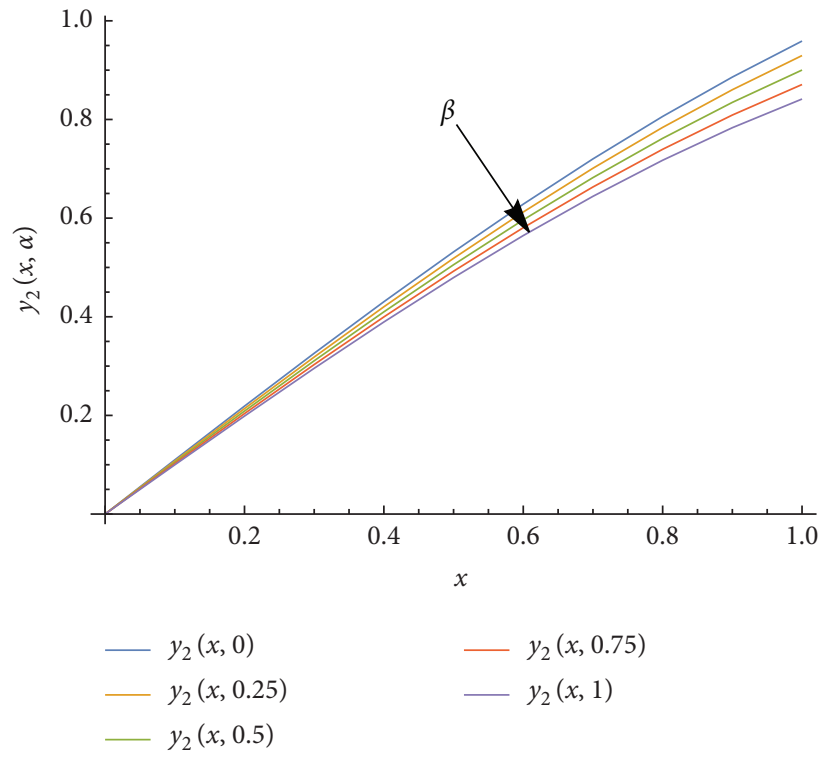

FIgURE 4: The approximate solution $y_{2}$ for $\beta=0,0.25,0.5,0.75,1$. 

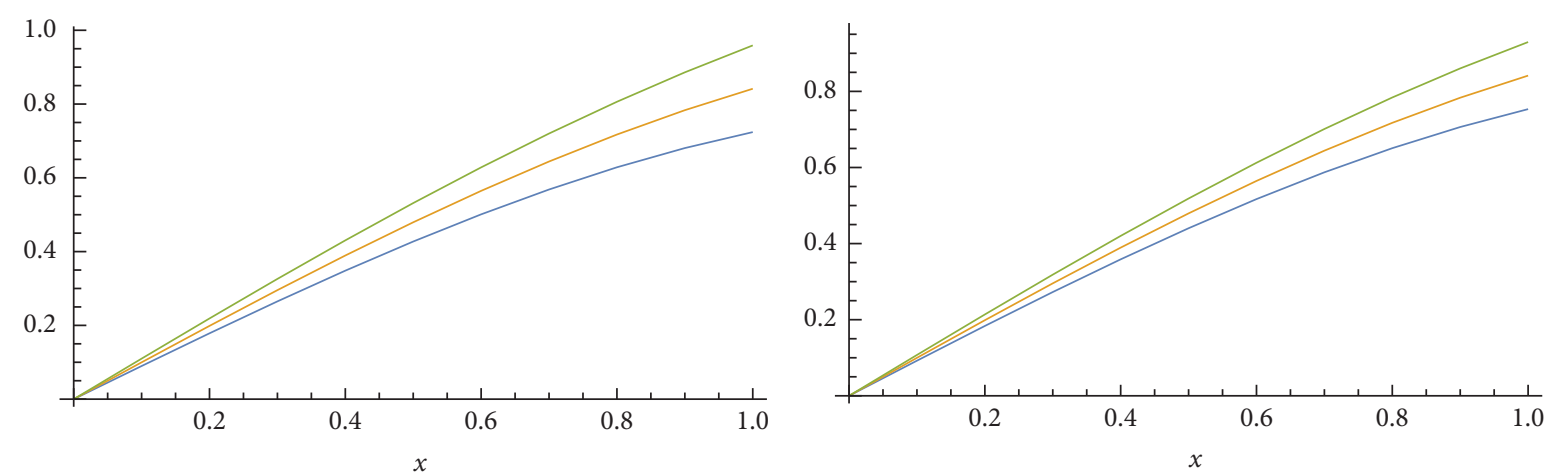

$$
\begin{aligned}
& -y_{1}(x, 0) \\
& -y_{c}(x) \\
& -y_{2}(x, 0)
\end{aligned}
$$

(a)

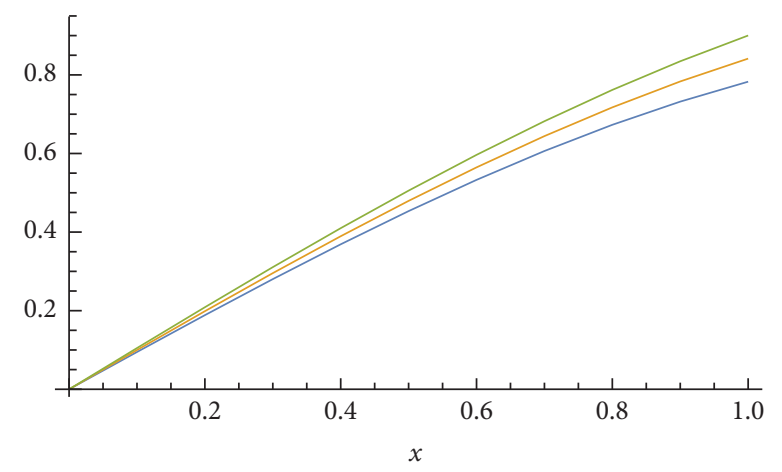

$$
\begin{aligned}
& -y_{1}(x, 0.25) \\
& -y_{c}(x) \\
& -y_{2}(x, 0.25)
\end{aligned}
$$

(b)

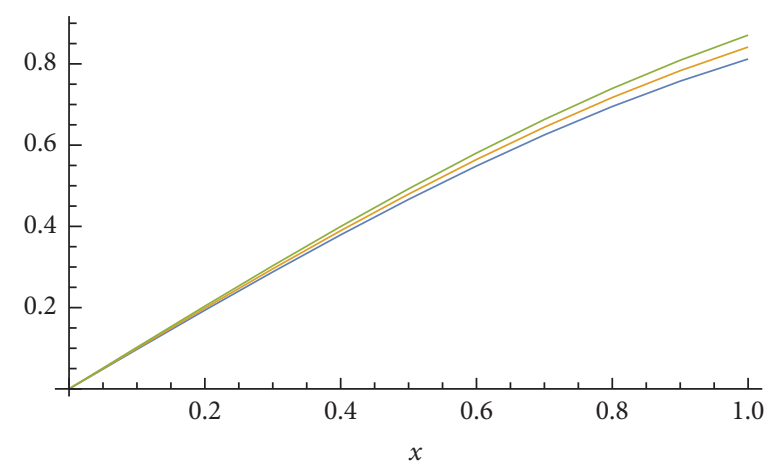

$$
\begin{aligned}
& -y_{1}(x, 0.5) \\
& -y_{c}(x) \\
& -y_{2}(x, 0.5)
\end{aligned}
$$

$$
\begin{aligned}
& -y_{1}(x, 0.75) \\
& -y_{c}(x) \\
& -y_{2}(x, 0.75)
\end{aligned}
$$

(c)

(d)

Figure 5: The approximate solution $y_{1}$ and $y_{2}$ and the crisp solution for $\beta=0,0.25,0.5,0.75$.

where $\hat{\gamma}_{\beta}=[\beta, 2-\beta], \hat{\theta}_{\beta}=[1+\beta, 3-\beta]$, and $h=0.01$. Let $y(x)=\left[y_{1}(x, \beta), y_{2}(x, \beta)\right]$ be a fuzzy solution and $y^{\prime}(x)=\left[y_{1}^{\prime}(x, \beta), y_{2}^{\prime}(x, \beta)\right]$. form:

Using $\beta$-level sets, the problem will have the following

$$
\begin{aligned}
{\left[y_{1}^{\prime \prime}(x, \beta), y_{2}^{\prime \prime}(x, \beta)\right] } & =-\left(\left[y_{1}(x, \beta), y_{2}(x, \beta)\right]\right)^{2}, \\
{\left[y_{1}(0, \beta), y_{2}(0, \beta)\right] } & =[\beta, 2-\beta], \\
{\left[y_{1}^{\prime}(0, \beta), y_{2}^{\prime}(0, \beta)\right] } & =[1+\beta, 3-\beta],
\end{aligned}
$$

where the exact solution is given by

$$
\left[\ln \left(\left(\beta e^{\beta}+e^{\beta}\right) x+e^{\beta}\right), \ln \left(\left(3 e^{2-\beta}-\beta e^{2-\beta}\right) x+e^{2-\beta}\right)\right] .
$$

In Tables 3 and 4 , we present the absolute error of results obtained by the current (HBM1) method and the ones obtained in [14].

Example 5. Consider the second-order fuzzy linear initial value problem

$$
\begin{gathered}
y^{\prime \prime}=y(x), \quad 0<x<1, \\
y(0)=\widehat{\theta}_{\beta}, y^{\prime}(0)=\widehat{\gamma}_{\beta},
\end{gathered}
$$

where $\widehat{\theta}_{\beta}=\widehat{\gamma}_{\beta}=[0.75+0.25 \beta, 1.125-0.125 \beta]$ and $h=0.1$. Let $y(x)=\left[y_{1}(x, \beta), y_{2}(x, \beta)\right]$ be a fuzzy solution.

By implementing the $\beta$-level sets, the problem will be 
TABLE 3: Absolute error for $y_{1,0.1}$.

\begin{tabular}{lcccc}
\hline \multicolumn{3}{c}{$y_{1,0.1}$} \\
$\beta$ & Error for $y_{1,0.1}$ & Error for $h_{1}$ HAM in $[14]$ & Error \\
& $2.42502 \times 10^{-10}$ & $1.53529 \times 10^{-7}$ & $3.98956 \times 10^{-8}$ & Error for $h_{2}$ HAM in [14] \\
\hline 0 & $5.79712 \times 10^{-10}$ & $4.51332 \times 10^{-7}$ & $3.91122 \times 10^{-10}$ & $1.59889 \times 10^{-10}$ \\
0.2 & $1.20466 \times 10^{-9}$ & $1.1207 \times 10^{-6}$ & $3.79328 \times 10^{-9}$ & $3.91122 \times 10^{-10}$ \\
0.4 & $2.25974 \times 10^{-9}$ & $2.4597 \times 10^{-6}$ & $4.47279 \times 10^{-8}$ & $3.79328 \times 10^{-9}$ \\
0.6 & $3.92067 \times 10^{-9}$ & $4.9128 \times 10^{-6}$ & $6.92457 \times 10^{-8}$ & $2.61947 \times 10^{-9}$ \\
0.8 & $6.39707 \times 10^{-9}$ & $9.10987 \times 10^{-6}$ & $1.53606 \times 10^{-7}$ & $6.69669 \times 10^{-8}$ \\
1 & & & & $1.11097 \times 10^{-8}$ \\
\hline
\end{tabular}

TABLE 4: Absolute error for $y_{2,0.1}$.

\begin{tabular}{lcccc}
\hline \multicolumn{3}{c}{$y_{2,0.1}$} \\
$\beta$ & Error for $y_{2,0.1}$ & Error for $h_{1}$ HAM in $[14]$ & Error \\
& $4.07084 \times 10^{-8}$ & $9.6735 \times 10^{-5}$ & $6.7076 \times 10^{-6}$ & Error for $h_{2}$ HAM in [14] \\
\hline 0 & $2.98335 \times 10^{-8}$ & $6.4822 \times 10^{-5}$ & $3.6619 \times 10^{-6}$ & $1.26440 \times 10^{-7}$ \\
0.2 & $2.13238 \times 10^{-8}$ & $4.2133 \times 10^{-5}$ & $1.8656 \times 10^{-6}$ & $9.18889 \times 10^{-8}$ \\
0.4 & $1.48046 \times 10^{-8}$ & $2.6432 \times 10^{-5}$ & $8.76047 \times 10^{-7}$ & $7.34552 \times 10^{-8}$ \\
0.6 & $9.93257 \times 10^{-9}$ & $1.5907 \times 10^{-5}$ & $3.76985 \times 10^{-7}$ & $3.52946 \times 10^{-8}$ \\
0.8 & $6.39707 \times 10^{-9}$ & $9.10987 \times 10^{-6}$ & $1.53606 \times 10^{-7}$ & $1.51728 \times 10^{-8}$ \\
1 & & & & $1.11097 \times 10^{-8}$ \\
\hline
\end{tabular}

$$
\begin{aligned}
{\left[y_{1}^{\prime \prime}(x, \beta), y_{2}^{\prime \prime}(x, \beta)\right] } & =\left[y_{1}(x, \beta), y_{2}(x, \beta)\right] \\
{\left[y_{1}(0, \beta), y_{2}(0, \beta)\right] } & =\left[y_{1}^{\prime}(0, \beta), y_{2}^{\prime}(0, \beta)\right]=[0.75+0.25 \beta, 1.125-0.125 \beta]
\end{aligned}
$$

where the exact solution is given by

$$
\left[(0.75+0.25 \beta) e^{x},(1.125-0.125 \beta) e^{x}\right]
$$

In Tables 5 and 6 , we present the absolute error of results obtained by the current (HBM1) method and the ones obtained in $[30,31]$ at $x=1$.

Example 6. Consider the second-order fuzzy linear initial value problem

$$
\begin{aligned}
{\left[y_{1}^{\prime \prime}(x, \beta), y_{2}^{\prime \prime}(x, \beta)\right] } & =x^{2} \odot\left[y_{1}^{\prime}(x, \beta), y_{2}^{\prime}(x, \beta)\right] \oplus 2 x \odot\left[y_{1}(x, \alpha), y_{2}(x, \alpha)\right] \oplus x \odot[2+2 \beta, 6-2 \beta], \\
{\left[y_{1}(0, \beta), y_{2}(0, \beta)\right] } & =[1+\beta, 3-\beta], \\
{\left[y_{1}^{\prime}(0, \beta), y_{2}^{\prime}(0, \beta)\right] } & =0,
\end{aligned}
$$

where the exact solution is given by

$$
\left[\left(2 e^{\left(x^{3} / 3\right)}-1\right)(1-\beta),\left(2 e^{\left(x^{3} / 3\right)}-1\right)(3-\beta)\right] .
$$


TABLE 5: Absolute error of $y_{1, \beta}$ at $x=1$ for $h=0.1$.

\begin{tabular}{|c|c|c|c|}
\hline & & $y_{1, \beta}$ & \\
\hline & & Error & \\
\hline$\beta$ & Error for $y_{1, \beta}$ & Error for method in [30] & Error for method in [31] \\
\hline 0 & $1.20 \times 10^{-16}$ & 0.003 & 0.003 \\
\hline 0.1 & $1.30 \times 10^{-12}$ & 0.003 & 0.003 \\
\hline 0.2 & $1.60 \times 10^{-12}$ & 0.003 & 0.003 \\
\hline 0.3 & $2.10 \times 10^{-12}$ & 0.01 & 0.01 \\
\hline 0.4 & $2.20 \times 10^{-12}$ & 0.01 & 0.01 \\
\hline 0.5 & $1.90 \times 10^{-12}$ & 0.008 & 0.004 \\
\hline 0.6 & $1.80 \times 10^{-12}$ & 0.004 & 0.004 \\
\hline 0.7 & $1.30 \times 10^{-12}$ & 0.004 & 0.003 \\
\hline 0.8 & $1.60 \times 10^{-12}$ & 0.01 & 0.002 \\
\hline 0.9 & $1.70 \times 10^{-12}$ & 0.01 & 0.01 \\
\hline 1 & $1.50 \times 10^{-12}$ & 0.004 & 0.004 \\
\hline
\end{tabular}

TABle 6: Absolute error of $y_{2, \beta}$ at $x=1$ for $h=0.1$.

\begin{tabular}{lccc}
\hline & & \multicolumn{1}{c}{$y_{2, \beta}$} & \multicolumn{1}{c}{$\begin{array}{c}\text { Error } \\
\beta\end{array}$} \\
& Error for $y_{2, \beta}$ & Error for method in [30] & Error for method in [31] \\
\hline 0 & $1.30 \times 10^{-16}$ & 0.002 & 0.002 \\
0.1 & $2.30 \times 10^{-12}$ & 0.001 & 0.001 \\
0.2 & $2.50 \times 10^{-12}$ & 0.001 & 0.001 \\
0.3 & $3.10 \times 10^{-12}$ & 0.005 & 0.005 \\
0.4 & $2.90 \times 10^{-12}$ & 0.01 & 0.01 \\
0.5 & $2.80 \times 10^{-12}$ & 0.001 & 0.005 \\
0.6 & $2.90 \times 10^{-12}$ & 0.01 & 0.01 \\
0.7 & $2.50 \times 10^{-12}$ & 0.01 & 0.001 \\
0.8 & $2.40 \times 10^{-12}$ & 0.01 & 0.005 \\
0.9 & $2.70 \times 10^{-12}$ & 0.004 & 0.01 \\
1 & $2.30 \times 10^{-12}$ & & 0.004 \\
\hline
\end{tabular}

TABLe 7: Absolute error of $y_{1, \beta}$ and $y_{2, \beta}$ at $x=1$ for $h=0.1$.

\begin{tabular}{|c|c|c|c|c|}
\hline \multicolumn{5}{|c|}{ Error } \\
\hline$\beta$ & Error for $y_{1, \beta}$ & Error for $y_{1, \beta}$ in [32] & Error for $y_{2, \beta}$ & Error for $y_{2, \beta}$ in [32] \\
\hline 0 & $1.20 \times 10^{-15}$ & $9.90 \times 10^{-8}$ & $2.30 \times 10^{-15}$ & $3.0 \times 10^{-7}$ \\
\hline 0.1 & $2.30 \times 10^{-12}$ & $1.10 \times 10^{-7}$ & $2.1 \times 10^{-12}$ & $2.90 \times 10^{-7}$ \\
\hline 0.2 & $2.40 \times 10^{-12}$ & $1.20 \times 10^{-7}$ & $3.1 \times 10^{-12}$ & $2.80 \times 10^{-7}$ \\
\hline 0.3 & $2.60 \times 10^{-12}$ & $1.30 \times 10^{-7}$ & $4.0 \times 10^{-12}$ & $2.70 \times 10^{-7}$ \\
\hline 0.4 & $2.90 \times 10^{-12}$ & $1.40 \times 10^{-7}$ & $4.3 \times 10^{-12}$ & $2.60 \times 10^{-7}$ \\
\hline 0.5 & $3.10 \times 10^{-12}$ & $1.50 \times 10^{-7}$ & $4.7 \times 10^{-12}$ & $2.50 \times 10^{-7}$ \\
\hline 0.6 & $3.20 \times 10^{-12}$ & $1.60 \times 10^{-7}$ & $5.0 \times 10^{-12}$ & $2.40 \times 10^{-7}$ \\
\hline 0.7 & $3.40 \times 10^{-12}$ & $1.70 \times 10^{-7}$ & $5.1 \times 10^{-12}$ & $2.30 \times 10^{-7}$ \\
\hline 0.8 & $3.50 \times 10^{-12}$ & $1.80 \times 10^{-7}$ & $5.3 \times 10^{-12}$ & $2.20 \times 10^{-7}$ \\
\hline 0.9 & $3.70 \times 10^{-12}$ & $1.90 \times 10^{-7}$ & $5.5 \times 10^{-12}$ & $2.10 \times 10^{-7}$ \\
\hline 1 & $4.0 \times 10^{-12}$ & $2.0 \times 10^{-7}$ & $5.7 \times 10^{-12}$ & $2.0 \times 10^{-7}$ \\
\hline
\end{tabular}

In Table 7, we present the absolute error of the results obtained by the current (HBM1) method and the ones obtained in [32] at $x=1$.

\section{Conclusion}

In this paper, we approximated the solution for fuzzy second-order initial value problems using HBM1. This method is characterized by high precision and stability. Besides, this method has an order of 3 which implies that it is consistent and convergent. Also, we drew the region of absolute stability. We solved four examples to show the efficiency and the accuracy of the proposed method. We note from the tables that our results are accurate even when we use only one off-step point. Moreover, our results with other results show that our results are more accurate. The computational 
cost is very reasonable since we used one off-step point. For researchers who are interested to get more accuracy, they can use two or three off-step points. The absolute error in this case will be almost zero. In addition, the proposed method is self-starter. We generate the approximate solutions using the initial conditions only. The results show that the proposed method is promising with fuzzy initial value problems. From the figures, we notice that the influence of the cut level on the lower solution is that as the cut level is increasing, the profile solution is increasing. Also, we notice that the influence of the cut level on the upper solution is that as the cut level is increasing, the profile solution is decreasing. Finally, we see that the crisp solution is bounded by lower and upper solutions and they become close to the crisp solution as the cut level becomes close to one.

\section{Data Availability}

All data are included within the article.

\section{Conflicts of Interest}

The authors declare that they have no conflicts of interest.

\section{References}

[1] T. Anake, D. Awoyemi, and A. O. Adesanya, "A one step method for the solution of general second order ordinary differential equations," International Journal of Science and Technology, vol. 2, no. 4, pp. 159-163, 2012.

[2] N. Z. Mukhtar, Z. A. Majid, F. Ismail, and M. Suleiman, "Numerical solution for solving second order ordinary differential equations using Block method," International Journal of Modern Physics: Conference Series, vol. 9, pp. 560-565, 2012.

[3] Y. Q. Hasan, "Solving singular initial value problems in the second-order ordinary differential equations," Journal of Applied Sciences, vol. 7, no. 17, pp. 2505-2508, 2007.

[4] K. Al-Khaled and M. N. Anwar, "Numerical comparison of methods for solving second-order ordinary initial value problems," Applied Mathematical Modelling, vol. 31, no. 2, pp. 292-301, 2007.

[5] M. J. Hossain, M. S. Alam, and M. B. Hossain, "A study on numerical solutions of second order initial value problems (IVP) for ordinary differential equations with fourth order and Butcher's fifth order Runge-Kutta methods," American Journal of Computational and Applied Mathematics, vol. 7, no. No. 5, pp. 129-137, 2017.

[6] H. Ramos, Z. Kalogiratou, T. Monovasilis, and T. Simos, "An optimized two-step hybrid block method for solving general second order initial-value problems," Numerical Algorithms, vol. 72, no. 4, pp. 1089-1102, 2016.

[7] B. Bede, "Fuzzy differential equations," Mathematics of Fuzzy Sets and Fuzzy Logic, vol. 295, pp. 171-191, 2013.

[8] M. Oberguggenberger and S. Pittschmann, "Differential equations with fuzzy parameters," Mathematical Modelling System, vol. 5, pp. 181-202, 1999.

[9] A. Bencsik, B. Bede, J. Tar, and J. Fodor, Fuzzy Differential Equations in Modeling Hydraulic Differential Servo Cylinders: Third Romanian-Hungarian Joint Symposium on Applied Computational Intelligence, SACI, Timisoara, Romania, 2006.
[10] L. C. Barros, R. C. Bassanezi, and P. A. Tonelli, "Fuzzy modelling in population dynamics," Ecological Modelling, vol. 128, no. 1, pp. 27-33, 2000.

[11] M. Syam, A. Alsuwaidi, A. Alneyadi, S. Al Refai, and S. Al Khaldi, "An implicit hybrid method for solving fractional Bagley-Torvik boundary value problem," Mathematics, vol. 6, no. 7, p. 109, 2018.

[12] M. A. Rufai and H. Ramos, "Numerical solution of secondorder singular problems arising in astrophysics by combining a pair of one-step hybrid block Nyström methods," Astrophysics and Space Science, vol. 365, pp. 1-13, 2020.

[13] M. I. Syam and M. Al-Refai, "A reliable method for first order delay equations based on the implicit hybrid method," Alexandria Engineering Journal, vol. 59, no. 4, pp. 2677-2681, 2020.

[14] A. F. Jameel, A. H. Shather, N. R. Anakira, A. K. Alomari, and A. Saaban, "Comparison for the approximate solution of the second-order fuzzy nonlinear differential equation with fuzzy initial conditions," Mathematics and Statistics, vol. 8, no. 5, pp. 527-534, 2020.

[15] Ö. Akın, T. Khaniyev, Ö. Oruç, and I. B. Türkşen, “An algorithm for the solution of second order fuzzy initial value problems," Expert Systems with Applications, vol. 40, no. 3, pp. 953-957, 2013.

[16] A. F. Jameel, M. Ghoreishi, and A. I. Ismail, "Approximate solution of high order fuzzy initial value problems," Journal of Uncertain Systems, vol. 8, no. 2, pp. 149-160, 2014.

[17] A. F. Jameel, N. R. Anakira, A. H. Shather, A. Saaban, and A. K. Alomari, "Numerical algorithm for solving second order nonlinear fuzzy initial value problems," International Journal of Electrical and Computer Engineering (IJECE), vol. 10, no. 6, pp. 6497-6506, 2020.

[18] A. Ahmadian, S. Salahshour, C. S. Chan, and D. Baleanu, "Numerical solutions of fuzzy differential equations by an efficient Runge-Kutta method with generalized differentiability," Fuzzy Sets and Systems, vol. 331, pp. 47-67, 2018.

[19] N. J. B. Pinto, E. Esmi, V. F. Wasques, and L. C. Barros, "Least square method with quasi linearly interactive fuzzy data: fitting an HIV dataset," in Fuzzy Techniques: Theory and ApplicationsSpringer, Berlin, Germany, 2019.

[20] E. Esmi, P. Sussner, G. Barroso Dias Ignácio, and L. Carvalho de Barros, "A parametrized sum of fuzzy numbers with applications to fuzzy initial value problems," Fuzzy Sets and Systems, vol. 331, pp. 85-104, 2018.

[21] V. F. Wasques, E. Esmi, L. C. Barros, and B. Bede, Comparison between Numerical Solutions of Fuzzy Initial-Value Problems via Interactive and Standard Arithmetics, Fuzzy Techniques: Theory and Applications, Springer International Publishing, Berlin, Germany, 2019.

[22] V. F. Wasques, E. Esmi, L. C. Barros, and P. Sussner, "The generalized fuzzy derivative is interactive," Information Sciences, vol. 519, pp. 93-109, 2020.

[23] E. Esmi, F. S. Pedro, L. C. De Barros, and W. Lodwick, "Fréchet derivative for linearly correlated fuzzy function," Information Sciences, vol. 435, pp. 150-160, 2018.

[24] A. G. Fatullayev, N. A. Gasilov, and Ş. Emrah Amrahov, "Numerical solution of linear inhomogeneous fuzzy delay differential equations," Fuzzy Optimization and Decision Making, vol. 18, no. 3, pp. 315-326, 2019.

[25] N. V. Hoa, "On the initial value problem for fuzzy differential equations of non-integer order \$\$ \alpha lin $(1,2) \$ \$$," Soft Computing, vol. 24, no. 2, pp. 935-954, 2020.

[26] N. V. Hoa and H. Vu, "A survey on the initial value problems of fuzzy implicit fractional differential equations," Fuzzy Sets and Systems, vol. 400, pp. 90-133, 2020. 
[27] N. Salamat, M. Mustahsan, and M. M. Saad Missen, "Switching point solution of second-order fuzzy differential equations using differential transformation method," Mathematics, vol. 7, no. 3, p. 231, 2019.

[28] U. M. Pirzada and D. C. Vakaskar, "Existence of Hukuhara differentiability of fuzzy-valued functions," Journal of the Indian Mathematical Society, vol. 84, no. 3-4, pp. 239-254, 2017.

[29] S. O. Fatunla, "A class of block methods for second order IVPs*:," International Journal of Computer Mathematics, vol. 55, no. 2, pp. 119-133, 1995.

[30] C. Duraisamy and B. Usha, "Another approach to solution of fuzzy differential equations by modified Euler's method," in 2010 International Conference on Communication and Computational Intelligence (INCOCCI), pp. 52-56, IEEE, Bhopal, India, December 2010.

[31] S. Tapaswini and S. Chakraverty, "A new approach to fuzzy initial value problem by improved Euler method," Fuzzy Information and Engineering, vol. 4, no. 3, pp. 293-312, 2012.

[32] M. Ghanbari, "Solution of the first order linear fuzzy differential equations by some reliable methods," Journal of Fuzzy Set Valued Analysis, vol. 2012, p. 20, 2012.

[33] A. Napoli and W. M. Abd-Elhameed, "An innovative harmonic numbers operational matrix method for solving initial value problems," Calcolo, vol. 54, no. 1, pp. 57-76, 2017.

[34] Y. H. Youssri, W. M. Abd-Elhameed, and M. Abdelhakem, "A robust spectral treatment of a class of initial value problems using modified Chebyshev polynomials," Mathematical Methods in the Applied Sciences, vol. 27, 2021.

[35] Ö. Akın, T. Khaniyev, O. Oruc, and I. B. Turksen, "Some possible fuzzy solutions for second order fuzzy initial value problems involving forcing terms," vol. 13, no. 2, pp. 239249, 2014.

[36] A. K. I. N. Ömer and O. R. U. Ç. Ömer, "A prey predator model with fuzzy initial values," Hacettepe Journal of Mathematics and Statistics, vol. 41, no. 3, pp. 387-395, 2012. 